OPEN ACCESS

Edited by:

Christopher Rensing,

Fujian Agriculture and Forestry

University, China

Reviewed by:

Xiaohua Su,

Chinese Academy of Forestry, China

Carla Caruso,

Università degli Studi della Tuscia, Italy

*Correspondence:

Qiang Zhuge

qzhuge@nifu.edu.cn

${ }^{\dagger}$ These authors have contributed equally to this work and share first authorship

Specialty section: This article was submitted to

Plant Microbe Interactions, a section of the journa

Frontiers in Microbiology

Received: 14 October 2019

Accepted: 17 January 2020

Published: 07 February 2020

Citation:

Wei H, Movahedi A, Xu C, Sun W. Wang P, Li D, Yin T and Zhuge $Q$ (2020) Characterization, Expression

Profiling, and Functional Analysis of PtDef, a Defensin-Encoding Gene

From Populus trichocarpa.

Front. Microbiol. 11:106.

doi: 10.3389/fmich.2020.00106

\section{Characterization, Expression Profiling, and Functional Analysis of PtDef, a Defensin-Encoding Gene From Populus trichocarpa}

\author{
Hui Wei ${ }^{1 \dagger}$, Ali Movahedi't, Chen Xu ${ }^{1,2+}$, Weibo Sun ${ }^{1}$, Pu Wang ${ }^{1}$, Dawei Li ${ }^{1}$, Tongming Yin ${ }^{1}$ \\ and Qiang Zhuge ${ }^{\text {** }}$
}

${ }^{1}$ Key Laboratory of Forest Genetics \& Biotechnology of Ministry of Education, Co-Innovation Center for Sustainable Forestry in Southern China, College of Biology and Environment, Nanjing Forestry University, Nanjing, China, ${ }^{2}$ Jiangsu Provincial Key Construction Laboratory of Special Biomass Resource Utilization, Nanjing Xiaozhuang University, Nanjing, China

PtDef cloned from Populus trichocarpa contained eight cysteine domains specific to defensins. Quantitative reverse-transcription polymerase chain reaction (qRT-PCR) analysis showed that PtDef was expressed in all tissues tested, with lower expression in leaves and higher expression in petioles, stems, and roots. Purified fused PtDef inhibited Aspergillus niger, Alternaria Nees, Mucor corymbifer, Marssonina populi, Rhizopus sp., and Neurospora crassa. PtDef also inhibited the growth of Escherichia coli by triggering autolysis. PtDef overexpression in Nanlin895 poplar (Populus $\times$ euramericana cv. Nanlin895) enhanced the level of resistance to Septotinia populiperda. qRT-PCR analysis also showed that the expression of 13 genes related to salicylic acid (SA) and jasmonic acid (JA) signal transduction differed between transgenic and wild-type (WT) poplars before and after inoculation, and that PR1-1 (12-72 h), NPR1-2, TGA1, and MYC2-1 expression was higher in transgenic poplars than in WT. During the hypersensitivity response $(\mathrm{HR})$, large amounts of $\mathrm{H}_{2} \mathrm{O}_{2}$ were produced by the poplar lines, particularly 12-24 h after inoculation; the rate and magnitude of the $\mathrm{H}_{2} \mathrm{O}_{2}$ concentration increase were greater in transgenic lines than in WT. Overall, our findings suggest that PtDef, a defensin-encoding gene of $P$. trichocarpa, could be used for genetic engineering of woody plants for enhanced disease resistance.

Keywords: JA, Populus trichocarpa, PtDef, defensin, Nanlin895 poplar, SA

\section{INTRODUCTION}

Plant defensins are antibacterial peptides that play an important role in plant immunity; they are the primary barrier to invasion by pathogenic bacteria (Ganz, 2003; Zhao et al., 2011). Animal and plant defensins evolved separately and have very different amino acid sequences and cystine connectivity (Shafee et al., 2016). Genes encoding plant defensins can be divided into those with higher and lower sequence homology; these two categories of defensins may have different biological activities (Stotz et al., 2009). Defensin-encoding genes have been identified in Gramineae (Bloch and Richardson, 1991), Cruciferae (Broekaert et al., 1995), gymnosperms (Brodelius and Xue, 1997; Do et al., 2004), and Cucurbitaceae (Da-Hui et al., 2007). Plant defensins are cysteine-rich cationic polypeptides 
of 45-50 amino acid residues $(\sim 5 \mathrm{kDa})$ and have antimicrobial activity (Vriens et al., 2014). Defensins have a tertiary structure comprising an $\alpha \beta(C S \alpha \beta)$ motif consisting of an $\alpha$-helix and three reverse parallel $\beta$-pleated sheets fixed by four disulfide bonds formed by eight cysteines (Yount and Yeaman, 2004). The expression of the genes encoding plant defensins differs among tissues and upon pathogen invasion (De Coninck et al., 2015; Pothana et al., 2019). For example, Arabidopsis PDF1.2 is a defense marker gene related to the jasmonic acid (JA) pathway and is upregulated by pathogens. Most plant defensins have antifungal activity (De Coninck et al., 2017), presumably mediated by interaction with specific sphingolipids on the fungal membrane (Thevissen et al., 2004; Cools et al., 2017). Phytodefensins inhibit protein synthesis (Méndez et al., 1996) and have alpha-amylase and protease activity (Pelegrini et al., 2008). Some plant defensins, but not all, bind to sphingolipids. Plant defensin activity is controlled by many different mechanisms (Parisi et al., 2018). Due to the functional diversity of plant defensin genes, they are widely used for genetic engineering. Transformation of an alfalfa defensin gene into potato enhanced potato resistance to Verticillium dahliae (Gao et al., 2001). Transformation of a radish defensin gene into tobacco improved tobacco resistance to Alternaria longicornis (Terras et al., 1993), and pea defensins inhibit pathogens and fungi in pea clip epidermis and vascular bundles (Almeida et al., 2002).

Plants defend against diseases using constitutive and induced mechanisms. Induced defense mechanisms play an important role in plant self-protection (Scheres and van der Putten, 2017). The hypersensitivity response (HR) is a type of cell death that evolved to suppress the replication of pathogens and mediates the resistance to plant disease (Fonseca and Mysore, 2019; Seifi and Shelp, 2019). Following pathogen invasion, the HR isolates the pathogen from the host plant, thus suppressing growth of the former (Gabriel and Rolfe, 1990). Cell death caused by the HR stimulates a defense response in adjacent tissues, leading to acquisition of systemic resistance. Therefore, the plant HR involves both cell death and the expression of resistance genes (Fonseca and Mysore, 2019; Seifi and Shelp, 2019). In the early stage of the HR, a large quantity of reactive oxygen species (ROS) is produced by plants, which further activates the HR (Fonseca and Mysore, 2019). The production of ROS, particularly hydroxyl radicals and $\mathrm{H}_{2} \mathrm{O}_{2}$, is an early cellular response to pathogens (Smirnoff and Arnaud, 2019), however, $\mathrm{H}_{2} \mathrm{O}_{2}$ can kill plant cells at high concentrations (Siddique et al., 2014). Antioxidant enzymes - peroxidase dismutase (SOD), peroxidase (POD), catalase (CAT), ascorbic acid peroxidase (APX), glutathione peroxidase (GPX), and glutathione-S-transferase (GST) - protect plants against damage by removing ROS. Phenylalanine ammonia lyase, chalcone synthase, and peroxidase are key enzymes in the biosynthesis of phenolic compounds and in lignin metabolism in plants (Vanholme et al., 2019). Most phenolics directly kill pathogenic bacteria, and lignin enhances the mechanical barrier of the plant cell wall. Upon development of disease, plants activate salicylic acid (SA), JA, ethylene (ET), and abscisic acid (ABA) signaling pathways, which mediate the response of plants to disease (Halitschke et al., 2004; Yang et al., 2018; Islam et al., 2019).
In the presence of disease, plants activate the expression of defense genes by regulating the synthesis of a variety of hormones. The transcription inhibitor, COI1 (coronatine insensitive 1)/JAZ (Jasmonate ZIM-domain), is important for the induction of plant disease resistance by JA and its derivatives. Jasmonoyl-L-isoleucine (JA-Ile) conjugates of JA and isoleucine are produced by plants in a diseased state. JAZ is degraded, which lifts the inhibition of transcription factor activity and induces the expression of JA signaling genes. MYC2 is an important transcription factor in this process, and links JAZ degradation to expression of genes related to JA signaling (Yan and Xie, 2015). $\mathrm{SA}$ induces the expression of pathogenesis-related protein $(P R)$, resulting in initiation of the HR. SA promotes the acquisition of resistance by accumulation of PR protein and ROS, and can be transported in plants (Cui et al., 2017; Svetaz et al., 2017). The non-expresser of physically related protein 1 (NPR1) is the dominant regulator of plant disease resistance. Maintenance of a low SA concentration in plant cells promotes accumulation of NPR1 and activation of TGA (WRKYS and TGACG sequencespecific binding proteins) transcription factors, which induces the expression of genes related to disease resistance (Fu et al., 2012). Previous studies have shown that TGA2, TGA5, and TGA6 inhibit the transcript level of $P R 1$ in the absence of SA, whereas TGA2, TGA5, and TGA6 are needed to induce PR1 in the presence of SA. In the absence of SA, TGA2 binds to the PR1 promoter, thereby inhibiting its transcription. The protein NIMIN1 can form ternary complexes with TGA2 through interaction with NPR1, at least in yeast. Transcriptional inhibition of TGA2 may be achieved by the interaction of NIMIN1 with a transcriptional co-repressor, without an upper body. It is conceivable that SA allows NPR1 to form different complexes with TGA2 and other TGA factors, such as TGA3, to activate PR1 transcription. The NIMIN1-NPR1-TGA2 complex dissociates in the presence of SA in yeast. Therefore, SA can separate NIMIN1 from the NPR1-TGA transcriptional complex, which may be helpful in activating the NPR1-TGA transcriptional complex (Seyfferth and Tsuda, 2014; Wang et al., 2015). JA and SA exert both synergistic and antagonistic effects on plant disease resistance (Yasuda et al., 2008).

The Poplar is among the most widely distributed and adaptable tree species. It is mainly distributed in temperate and cold-temperate regions of the northern hemisphere (latitude 22$70^{\circ} \mathrm{N}$ ), including China, Russia, Canada, United States, Italy, and France. The Poplar has been used in studies of the genome of perennial plants due to the availability of its genome sequence (Tuskan et al., 2006). Genetic engineering can be applied to improve poplar. Septotinia populerda was first discovered as a poplar disease in 1950 (Waterman and Cash, 1950). Following infection by $S$. populerda, brown spots form in infected leaf parts, gradually becoming gray-white holes. If the poplars are damaged year after year, the tree will weaken, enhancing conditions for disease occurrence via weak pathogens. Poplar is an important industrial plantation species worldwide; infection by S. populerda seriously affects poplar production, in turn significantly reducing the economic and ecological benefits of poplar (Kaul et al., 2010; Wingfield et al., 2015). Plant defensins have antimicrobial activity and play an important role in the stress response, so they are a 
focus in the research of plant diseases. In this study, we cloned the PtDef defensin-encoding gene from poplar. We investigated the tissue-specificity of, and the effect of a variety of stresses on, the expression of PtDef by quantitative RT-PCR. Also, PtDef was produced in a prokaryotic system, purified, and its activity evaluated in vitro. PtDef induced autolysis of Escherichia coli BL21 (DE3) and activated expression of a two-component system (TCS). Moreover, Nanlin 895 poplar (Populus $\times$ euramericana cv. Nanlin 895) was transformed with PtDef by the Agrobacterium method. The transgenic poplar exhibited greater resistance to $S$. populiperda than the wild-type (WT) poplar. Furthermore, the expression of $P R 1, N P R 1$, and TGA (related to SA signaling) and of $M Y C 2, J A Z$, and COI1 (related to JA signaling) was evaluated in transgenic and WT poplars infected with $S$. populiperda. We explored the plant-hormone signal transduction mechanisms in poplar that mediate the resistance to $S$. populiperda, and our results will facilitate the screening for and breeding of diseaseresistant poplar varieties.

\section{RESULTS}

\section{Cloning and Sequence Analysis of PtDef}

We cloned PtDef (XP_002325735.1) from P. trichocarpa (Tuskan et al., 2006). PtDef had a 225-bp open reading frame (ORF) that encoded a 74 amino acid polypeptide (Supplementary Figure S1). The predicted molecular weight and isoelectric point of PtDef were $8.13 \mathrm{kDa}$ and 8.98 , respectively. A comparison of the amino acid sequence of PtDef with that of other defensins indicated the presence of eight invariant residues of cysteine (Supplementary Figure S2). The degree of conservation was higher at the $\mathrm{C}$-terminus than at the $\mathrm{N}$-terminus (Supplementary Figure S2). The $\mathrm{N}$-terminus of PtDef has a 27 amino acid signal peptide (Figure 1).

The eight conserved Cys residues in PtDef form four intrachain disulfide bonds: Cys1-Cys8, Cys2-Cys5, Cys3-Cys6, and Cys4-Cys7 (Supplementary Figure S3). The Cys1-Cys8 and Cys2-Cys5 bonds are characteristic of plant defensins

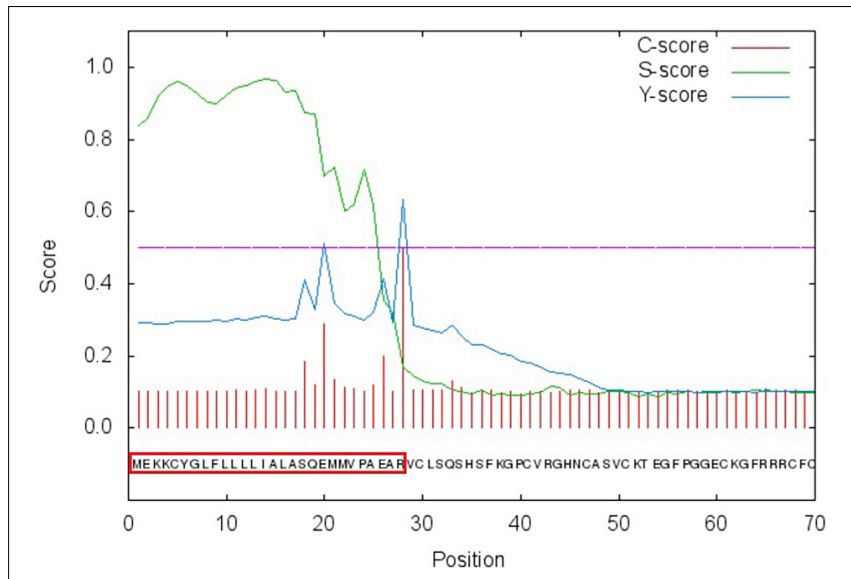

FIGURE 1 | Signal peptide analysis of PtDef. Red box, signal peptide.
(Selsted and Ouellette, 2005). A defensin phylogenetic tree constructed using MEGA5 software showed that PtDef was closely related to a defensin of Ricinus communis (XP_025012078.1) (Supplementary Figure S4). In R. communis (XP_025012078.1), the function of RcDef was annotated as gamma-thionin, indicating that PtDef may have similar activity.

\section{Tissue-Specificity and Induction of the Expression of PtDef}

The expression of PtDef was detected by qRT-PCR in all tissues tested, with the highest expression in petioles, followed by stems, roots, and leaves, particularly in young leaves (Figure 2A).

Treatment with $200 \mu \mathrm{M}$ ABA induced the expression of PtDef (Figure 2B); expression peaked at $3 \mathrm{~h}$ and subsequently decreased slightly to a level above background. Treatment with $200 \mu \mathrm{M}$ JA significantly increased PtDef expression at $6 \mathrm{~h}$ with a peak at $8 \mathrm{~h}$ (Figure 2C), followed by a gradual decrease to a level significantly higher than the control upon treatment, as indicated by leakage of cell contents. Treatment with $200 \mu \mathrm{M}$ SA at $1 \mathrm{~h}$ resulted in no statistically significant increase in PtDef expression, followed by a decrease to a low level at 3-48 h (Figure 2D). Treatment with $2 \mathrm{mM} \mathrm{H}_{2} \mathrm{O}_{2}$ increased PtDef expression at $1 \mathrm{~h}$, followed by a gradual increase to a peak at $12 \mathrm{~h}$ and a decrease at 24-48 $\mathrm{h}$ to a level significantly higher than the control (Figure 2E). In the presence of $200 \mathrm{mM} \mathrm{NaCl}$, PtDef expression peaked at $8 \mathrm{~h}$, decreased, and subsequently increased to a second peak at $48 \mathrm{~h}$ (Figure 2F). Under 10\% PEG $_{6000}$ treatment, PtDef expression increased gradually at days 1-4, decreased slightly at day 5 to a level above that of the control, and subsequently increased to a peak at day 6 (Figure 2G). Under cold treatment, PtDef expression peaked at day 4 , subsequently decreased slightly to a level above that of the control and reached a higher peak at day 7 (Figure 2H). Under wound stress, PtDef expression increased gradually beginning at $1 \mathrm{~h}$ to a peak at $9 \mathrm{~h}$, followed by a gradual decrease (Figure 2I). Following inoculation with S. populiperda, PtDef expression increased significantly at $12 \mathrm{~h}$ and then decreased (Figure 2J). These results suggest that PtDef responds to abiotic and biotic stresses and is involved in a variety of physiological processes.

\section{Prokaryotic Expression and Purification of Recombinant PtDef}

The ORF of PtDef was ligated into PET-32a using the NotI and BamHI sites (Figure 3A). Upon SDS-PAGE analysis, IPTGinduced, but not non-induced, E. coli BL21 (DE3) yielded a band for the Trx-PtDef fusion protein (Figure 3B). SDS-PAGE analysis showed that Trx-PtDef was present in the sediment (Figure 3C, Lane 2) and supernatant (Figure 3C, Lane 3). The presence of supernatant simplified PtDef purification by obviating the need for denaturation and renaturation.

Purification of Trx-PtDef by Ni-IDA resin affinity chromatography (Figure 3D) yielded an outflow peak and an elution peak. Upon SDS-PAGE analysis, a clear band was detected in the effluent and eluent liquid; the protein within this band was identified as Trx-PtDef by western blotting (Figure 3E). 


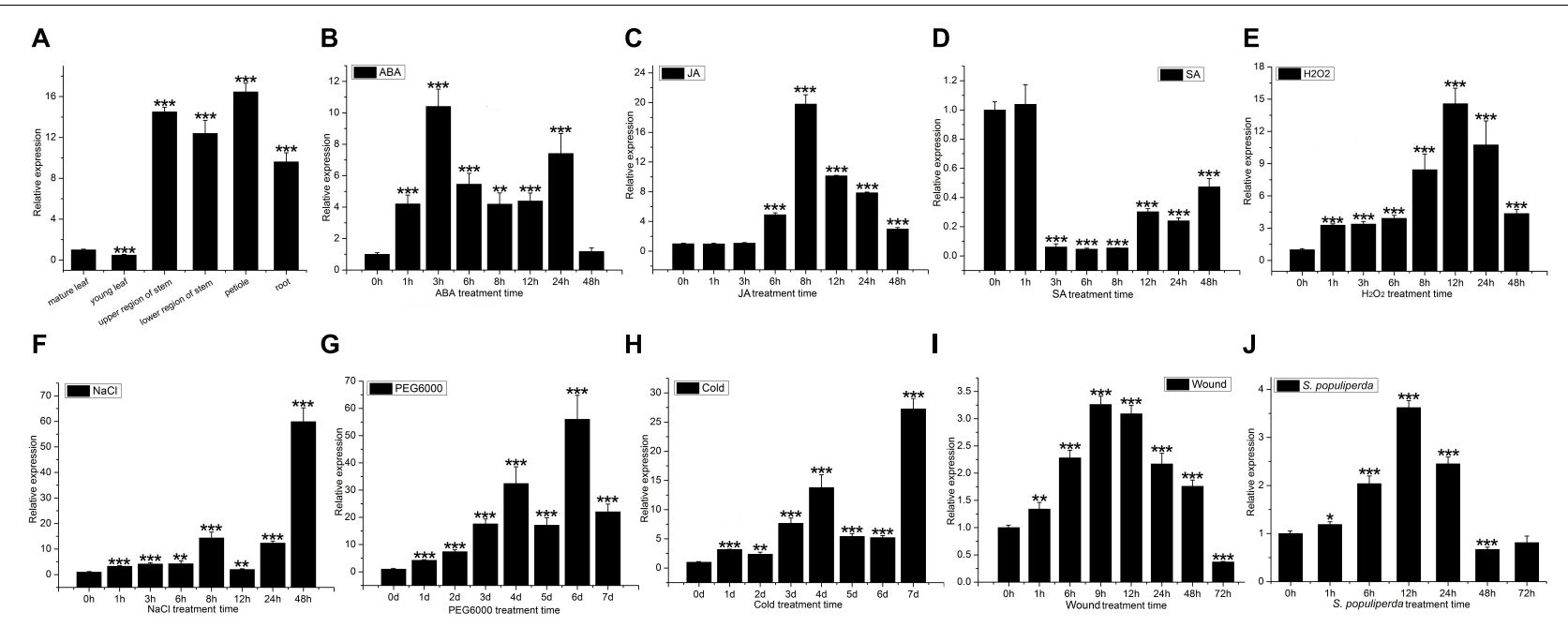

FIGURE 2 | PtDef expression in poplar tissues and under abiotic and biotic stresses. (A) PtDef expression in mature leaves, young leaves, upper region of stems, lower region of stems, petioles, and roots. Student's $t$-test, ${ }^{\star \star \star} P<0.001$ compared to the value in mature leaves. Effects on PtDef expression of (B) abscisic acid (ABA), (C) jasmonic acid (JA), (D) salicylic acid (SA), (E) hydrogen peroxide $\left(\mathrm{H}_{2} \mathrm{O}_{2}\right)$, (F) NaCl, (G) PEG $\mathrm{F}_{6000}$, (H) cold, (I) wounding, and (J) S. populiperda. Values are means \pm standard deviation (SD) of three biological replicates. Student's $t$-test, ${ }^{\star \star \star} P<0.001,{ }^{\star \star} P<0.01$, and ${ }^{\star} P<0.05$ relative to $0 \mathrm{~h}$.

\section{Antifungal Activity of PtDef}

The antifungal activity of purified recombinant PtDef was assayed in vitro (Figure 4) using the agarose diffusion method. Recombinant PtDef inhibited the growth of A. niger, A. Nees, M. corymbifer, M. populi, Rhizopus sp., and N. crassa, while the growth of the control was normal. The antifungal activity of PtDef exhibited dose dependency and differed among the fungal species tested.

\section{IPTG-Induced Production of PtDef Inhibits the Growth of $E$. coli}

The addition of IPTG for 2-4 h inhibited the growth of E. coli BL21 (DE3). The $\mathrm{OD}_{600}$ value of E. coli BL21 (DE3) cultures was stable 3-4 $\mathrm{h}$ after the addition of IPTG. In contrast, the growth of E. coli BL21 (DE3) in the absence of IPTG (control) was normal. Therefore, IPTG-induced production of PtDef inhibits growth of E. coli BL21 (DE3) (Supplementary Figure S5A).

To investigate the mechanism by which PtDef inhibits E. coli BL21 (DE3), we performed a transcriptome analysis. According to the paradigm of promotion by antibiotics of bacterial autolysis (Supplementary Figure S5B; Kohanski et al., 2007), antibiotics modulate the $\mathrm{NAD}(\mathrm{H})$ level by activating the electron transport chain, triggering superoxide production. Superoxide disrupts the iron oxidation balance, leading to the formation of hydroxyl radicals; the destruction of DNA, proteins, and lipids; and ultimately cell death. The expression of 13 of the 26 genes involved in the TCA cycle was altered; seven (fumC, sdhD, sdhC, gltA, sdhB, sdhA, and $s u c A$ ) were upregulated and six ( $a c n A, y d b K, f r d C, f r d D, f r d A$, and $f r d B$ ) were downregulated (Supplementary Figure S6A). Additionally, the expression of 16 of the 41 genes involved in oxidative phosphorylation was altered; six ( $c y o A, c y o E, s d h D, s d h C, s d h B$, and $s d h A)$ were upregulated and 10 (nuoF, nuoE, nuoC, ppa, appC, frdC, appB, frdD, frdA, and $f r d B$ ) were downregulated compared with the control (Supplementary Figure S6B). Moreover, the expression of seven genes related to RNA degradation was altered; four $(r h l B$, $h f q$, rho, and $r p p H$ ) were upregulated and three ( $g r o L$, eno, and $r n e$ ) were downregulated (Supplementary Figure S7A). The expression of 26 genes $(r p l C, r p l D, r p l W, r p l B, r p s S, r p l V, r p s C, r p l P, r p s M$, $r p s K, r p l Y, r p l F, r p s D, r p s B, r p s J, r p l X, r p l M, r p s H, r p s N, r p s E$, $r p l R, r p l K, r p l A, r p s F, r p s G$, and $r p s L$ ) related to the ribosome was downregulated (Supplementary Figure S7B). The expression of two genes related to DNA replication was altered; dnaE was downregulated and $r n h A$ was upregulated (Supplementary Figure S7C). Some bactericidal antibiotics disrupt bacterial respiration by blocking the entry of pyruvate into the TCA cycle, which slows growth. We found that the expression of 10 genes related to glycolysis/gluconeogenesis (nuoF, nuoE, nuoC, $p p a, a p p C, f r d C, a p p B, f r d D, f r d A$, and $f r d B$ ) was downregulated (Supplementary Figure S8A). Also, $p y k A$, the product of which mediates the production of pyruvate in the glycolytic pathway, was downregulated, as was that of the gene encoding pyruvate kinase $(p y k F)$, indicating suppression of the generation of lactate from pyruvate. Finally, $f r d A, f r d B, f r d C$, and $f r d D$ were downregulated, and the transcript levels of genes for succinate acid under fermentation were downregulated (Supplementary Figure S8B). Based on the above, we hypothesized that PtDef induces bacterial autolysis.

\section{Transcriptome Sequencing Analysis}

Using Illumina second-generation high-throughput sequencing we generated a total of $24,849,094$ raw read pairs. After quality control, 24,340,267 clean read pairs were obtained. Analysis using Bowtie2 and Rockhopper software resulted in the identification of 639 differentially expressed genes (DEGs). Compared with the control, 359 DEGs were upregulated and 280 were downregulated in the experimental group (Figure 5). The 639 
A

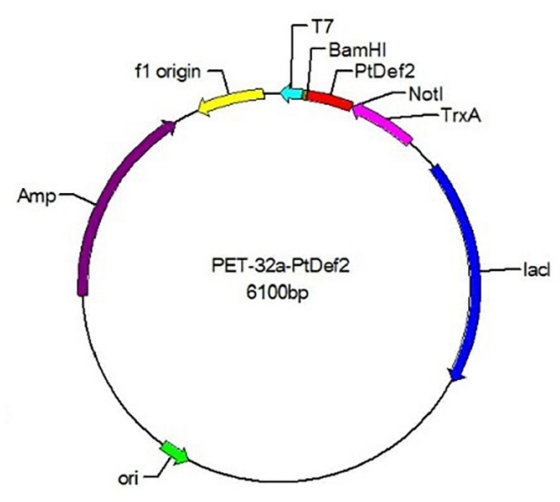

D

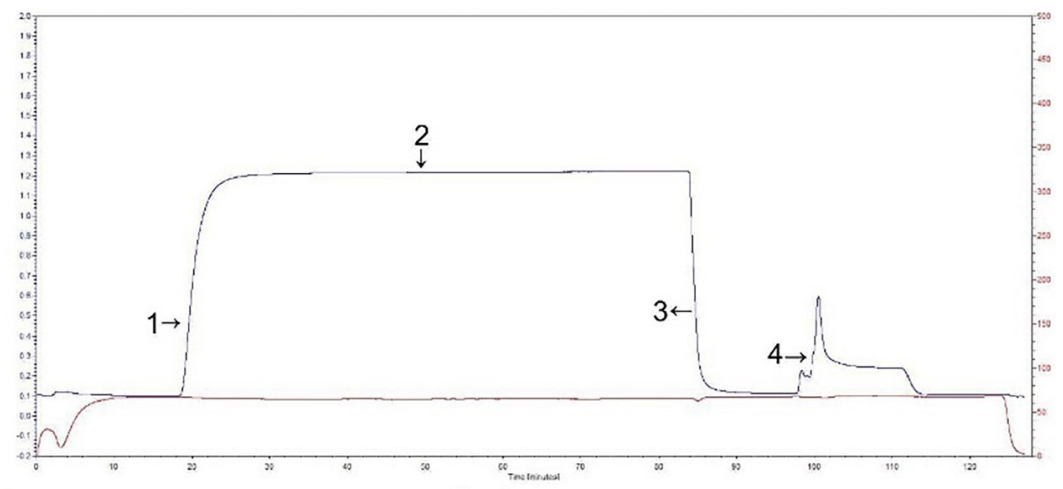

B

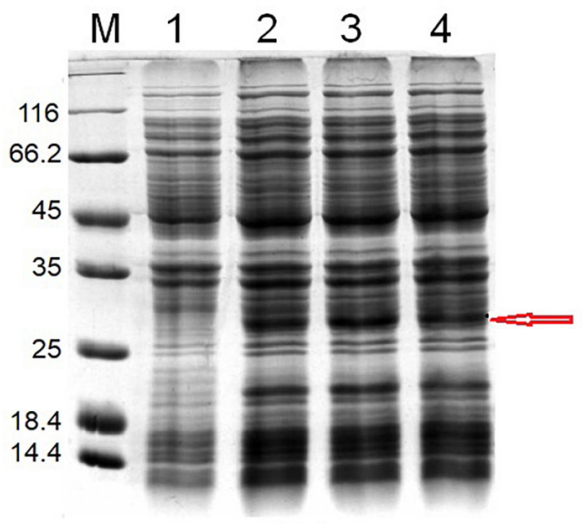

C

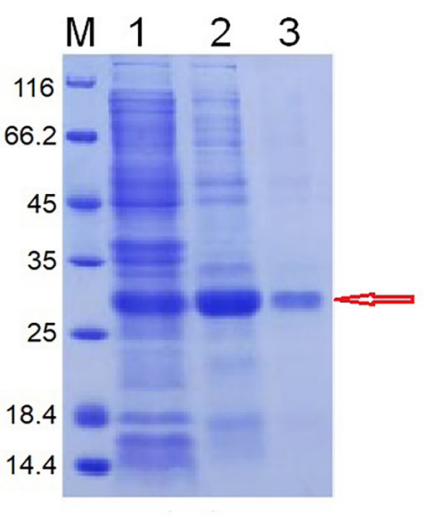

E

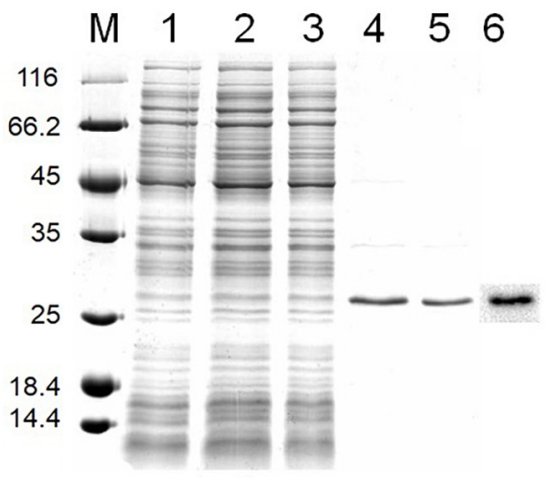

FIGURE 3 | Prokaryotic expression and purification. (A) Construction of the expression vector PET-32a-PtDef. PtDef was inserted into BamHI and Notl restriction sites. (B) Analysis of the PtDef fusion protein by 12\% SDS-PAGE. Lane M, molecular mass marker; Lane 1, negative control (non-induction); Lanes 2-4, colonies 1-3 (induction); red arrow, PtDef fusion protein. (C) Analysis of PtDef fusion protein supernatant and sediment by 12\% SDS-PAGE. Lane M, molecular mass marker: Lane 1, induction; Lane 2, sediment; Lane 3, supernatant; red arrow, PtDef fusion protein. (D) Ni-IDA affinity chromatography of the Trx-PtDef fusion protein using LP Data View. Lanes 1 and 2, flow-through; Lane 3, wash; Lane 4, elution. (E) Purification and western blotting of PtDef fusion protein. Lane M, molecular weight marker; Lanes 1 and 2, flow-through; Lane 3, wash; Lanes 4 and 5, elution; Lane 6, western blot of PtDef using a monoclonal antibody against the $6 \times$ His tag.

unigenes clustered into three expression patterns according to their expression levels in the control and experimental groups (Supplementary Figure S9).

The 639 DEGS were divided into three major GO categories (30 functional groups): biological processes, cellular components, and molecular functions. The three most highly enriched functional groups were cellular metabolic process, biosynthetic process, and cellular biosynthetic process (Supplementary Figure S9). The top 20 enriched pathways (Supplementary Figure S10) included biosynthesis of secondary metabolites, biosynthesis of antibiotics, and microbial metabolism in diverse environments.

The expression of 10 genes related to TCS (narZ, narY, pstS, hyaC, appC, frdC, appB, frdD, frdA, and $\operatorname{frdB}$ ) was downregulated and that of 12 genes (gltI, $\operatorname{arcA}$, evgA, narP, crp, ompF, NA, fepA, glnL, glnA, baeR, and zraP) was upregulated (Supplementary Table S2). Bacteria survive in hostile environments by regulating their gene pool. TCS is involved in bacterial adaptation to changes in the environment, as well as antibiotic resistance and virulence (Stock et al., 2000;
Stephenson and Hoch, 2002). The SOS reaction maintains bacterial growth after damage and is important for sensing and transmitting antibiotic resistance and virulence factors (Beceiro et al., 2013). Based on the transcriptome data, we conclude that PtDef induced by IPTG inhibited E. coli, but that E. coli adopted strategies such as TCS to regulate bacteria to adapt to the adverse environment. The expression of genes related to the SOS response, such as lexA and RecA (Baharoglu et al., 2010), was not altered. During bacterial drug resistance, the SOS response regulated by $\operatorname{RecA}$ is an important pathway for drug resistance (Baharoglu et al., 2010). Together, these findings indicate that TCS is triggered, but that the SOS response fails to trigger, when E. coli is maintained in an adverse environment where PtDef inhibits its growth.

\section{Identification of Transgenic Nanlin895 Lines}

We screened 10 transgenic poplar lines throughout the differentiation, bud elongation, and rooting stages. PCR 

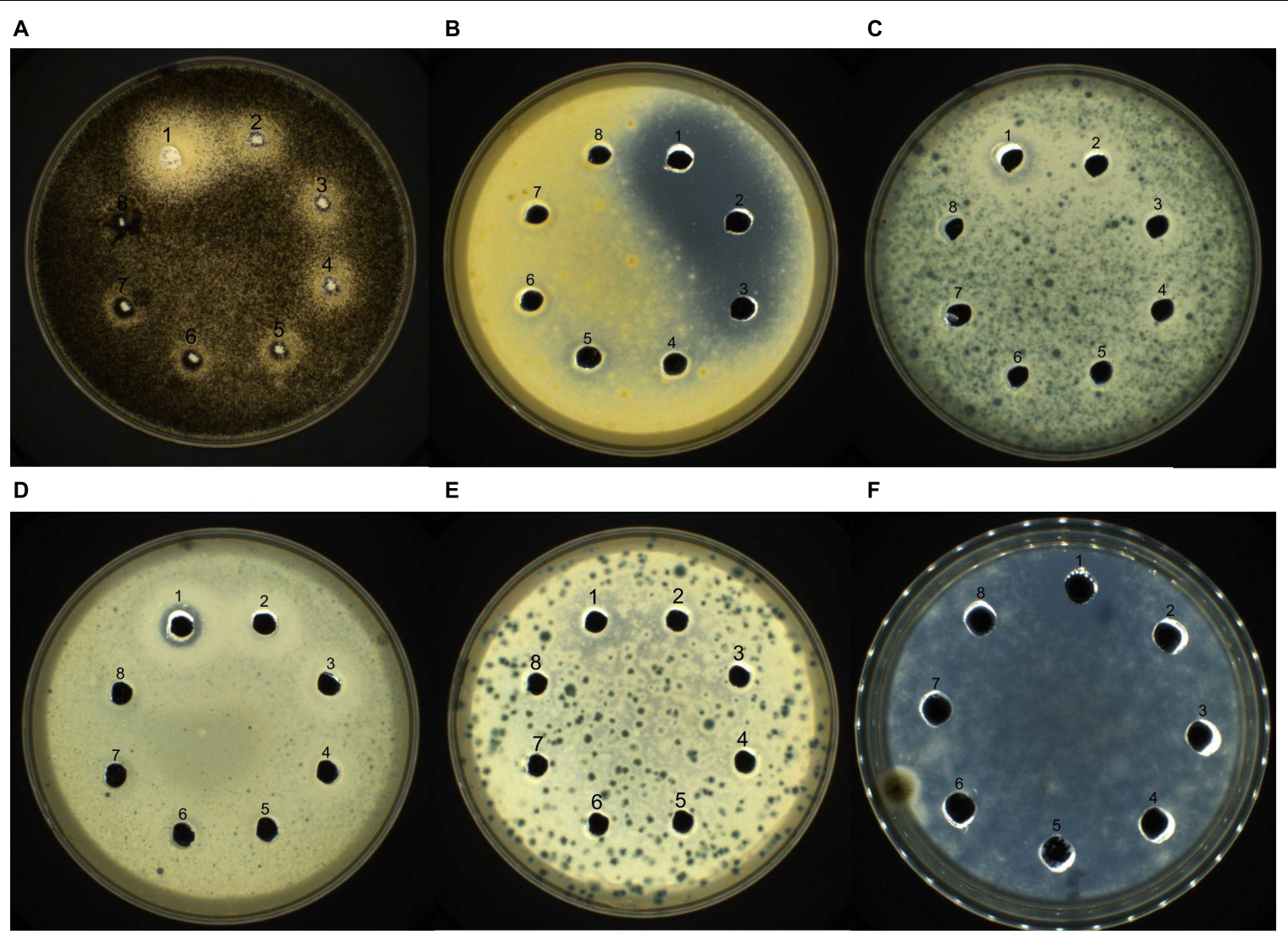

FIGURE 4 | Bioactivity of PtDef against (A) Aspergillus niger, (B) Alternaria Nees, (C) Mucor corymbifer, (D) Marssonina populi, (E) Rhizopus sp., and (F) Neurospora crassa. Lanes 1-7, purified Trx-PtDef fusion protein (40, 20, 10, 5, 2.5, 1.25, and $0.625 \mu \mathrm{g} / \mathrm{mll}$ ); Lane 8, 0.9\% NaCl (negative control). Each well was loaded with $80 \mu$ l of sample.

using PtDef-F and PtDef-R specifically amplified 225-bp bands in the transgenic lines, whereas WT poplars had a much larger band (Supplementary Figure S11A). Using $35 \mathrm{~S}$ and PtDef-R as forward and reverse primers, PCR yielded specific bands from transgenic but not WT poplars (Supplementary Figure S11B), indicating genome integration of PtDef. qRT-PCR confirmed that expression of PtDef was 2 - to 4-fold higher in transgenic poplar than in WT poplar (Supplementary Figure S11C).

\section{Disease Tolerance of Transgenic Nanlin895 Poplar}

We evaluated the resistance to $S$. populiperda infection of the transgenic (Trans1 and Trans2) and WT poplars. Plaques grew more rapidly on WT poplar leaves than on transgenic poplar leaves at 2-4 days after infection (Figure 6A). On days 2 and 4, spots covered $24.33 \pm 3.21 \%$ and $14.67 \pm 2.08 \%$ (Trans 1 ) or $9.67 \pm 1.53 \%$ (Trans 2), and $48.33 \pm 7.64 \%$ and $30.33 \pm 3.06 \%$ (Trans 1 ) or $23.67 \pm 4.73 \%$ (Trans 1), of the surface of WT and transgenic poplar leaves, respectively (Figure 6B).

Evaluation of the damage to poplar leaves caused by $S$. populiperda revealed that little damage had been inflicted on the cells of transgenic poplar on day 4 , while the cells of WT poplar leaves showed considerable damage at day 4 by microscopy (Figure 7A). The generation time of $S$. populiperda was shorter on the leaves of the WT poplar than the transgenic poplar (Figure 7B). Therefore, overexpression of PtDef in Nanlin895 poplar enhanced resistance to $S$. populiperda.

\section{Effect of S. populiperda on the Expression of Genes Related to SA and JA Pathways}

The expression of PR1-1 was slightly lower in transgenic poplar than in WT at $0-12 \mathrm{~h}$; at $72 \mathrm{~h}$, the opposite trend was observed, such that PR1-1 expression was 51.5-fold upregulated in transgenic plants and 6.4-fold upregulated in WT (Figure 8A). PR1-2 expression was significantly lower at $12-24 \mathrm{~h}$ in transgenic poplar than in WT. However, PR1-2 expression showed the opposite trend at $72 \mathrm{~h}$ (Figure $\mathbf{8 B}$ ). TGA1 expression was higher in the transgenic poplar than in WT at $0-72 \mathrm{~h}$ (Figure 8C). In transgenic poplar, TGA2 expression was higher at $0 \mathrm{~h}$ and $48 \mathrm{~h}$ (Figure 8D). NPR1-1 expression was higher in transgenic poplar than in WT under pre-inoculation, and lower than in WT poplar at $72 \mathrm{~h}$ (Figure 8E). NPR1-2 expression was higher in the transgenic line than in WT at 0-48 h (Figure 8F). 


\section{Def vs Control}

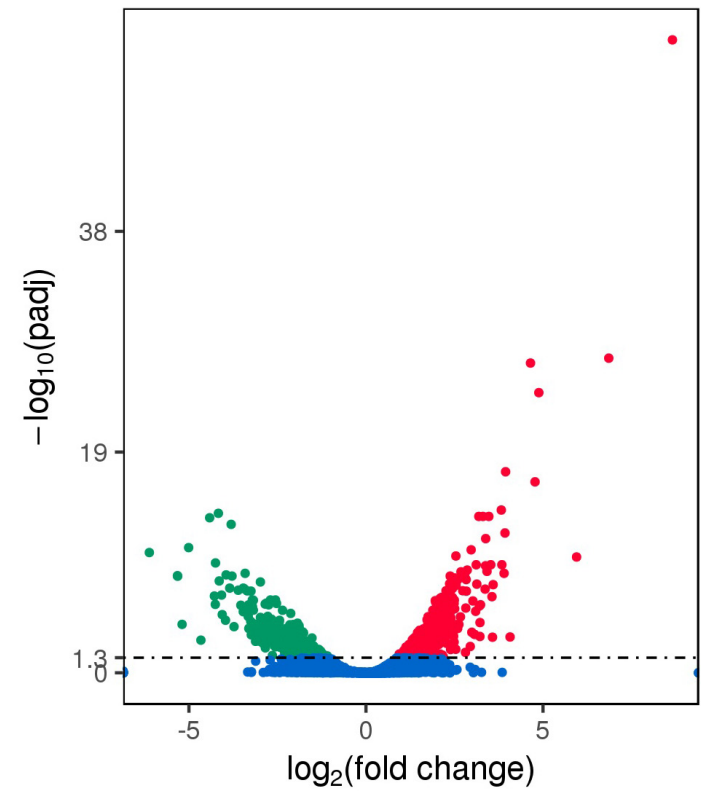

Differential Expressed Genes ( 639 )

- up regulated: 359

- down regulated: 280

FIGURE 5 | Volcano map of differentially expressed genes (DEGs). The abscissa represents the fold change in expression and the ordinate represents the significance of the change in gene expression. Smaller corrected $P$ values indicate larger values of $-\log (P)$, i.e., greater statistical significance.
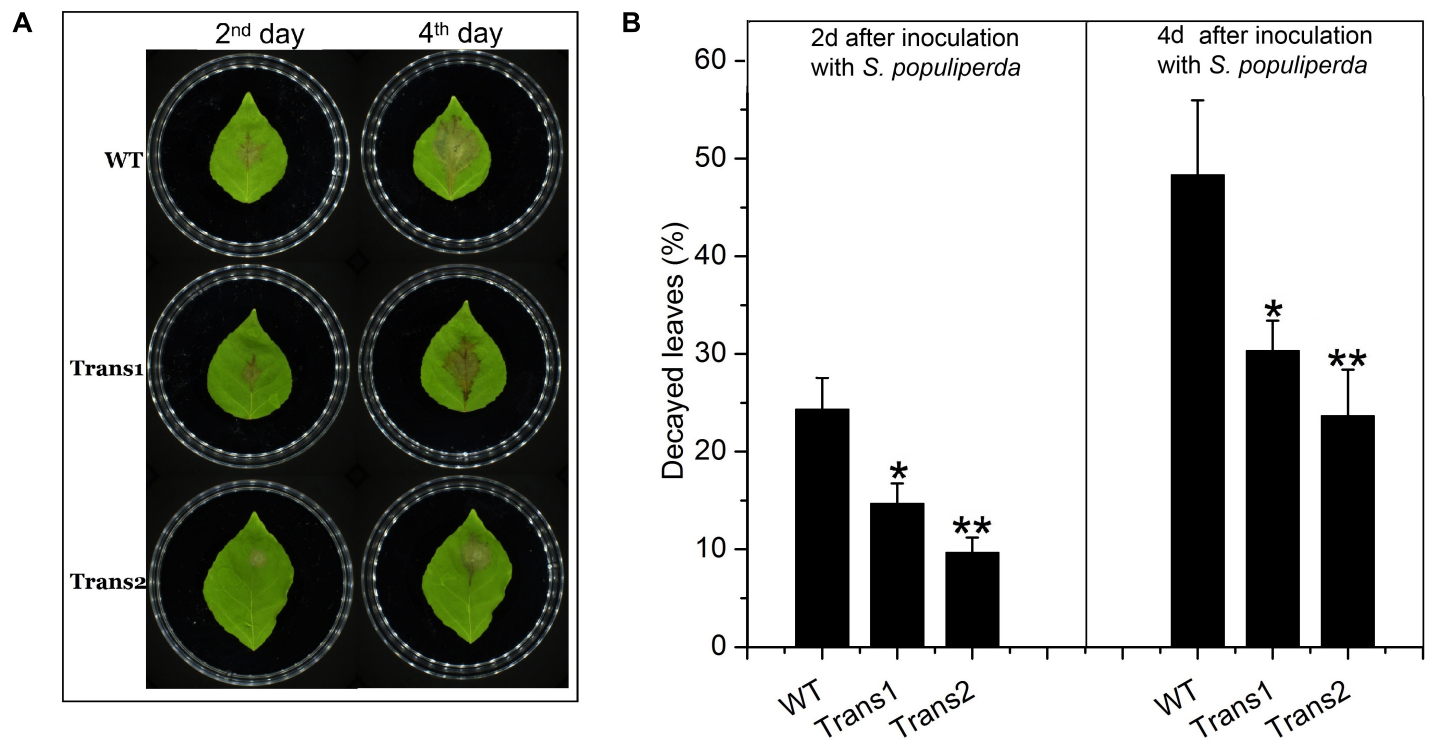

FIGURE 6 | Overexpression of PtDef enhances resistance to S. populiperda. (A) Transgenic poplar overexpressing PtDef showed fewer lesions than WT poplar following inoculation with S. populiperda. (B) Diseased area of transgenic and WT poplar following inoculation with $S$. populiperda. Values are means \pm SD of three biological replicates. Student's $t$-test, ${ }^{* \star} P<0.01$ relative to $0 \mathrm{~h}$.

COI1-1 expression was lower in transgenic poplar than in WT before inoculation with $S$. populiperda. Post-inoculation, COI1-1 expression was higher in transgenic poplar than in WT at 12-48 h (Figure 8G). COI1-2 expression was higher in the transgenic poplar than in WT at $12-48 \mathrm{~h}$, except at 0 and $72 \mathrm{~h}$ (Figure $\mathbf{8 H}$ ). Post-inoculation, JAZ1 expression was lower in transgenic poplar than in WT at $24 \mathrm{~h}$ (Figure 8I). The expression of JAZ2 in transgenic poplar was markedly lower than that in WT pre-inoculation. JAZ2 expression in WT was lower following inoculation with $S$. populiperda, whereas in transgenic poplar JAZ2 expression was higher at $12 \mathrm{~h}$ (Figure 8J). The expression of MYC2-1 was higher in transgenic 

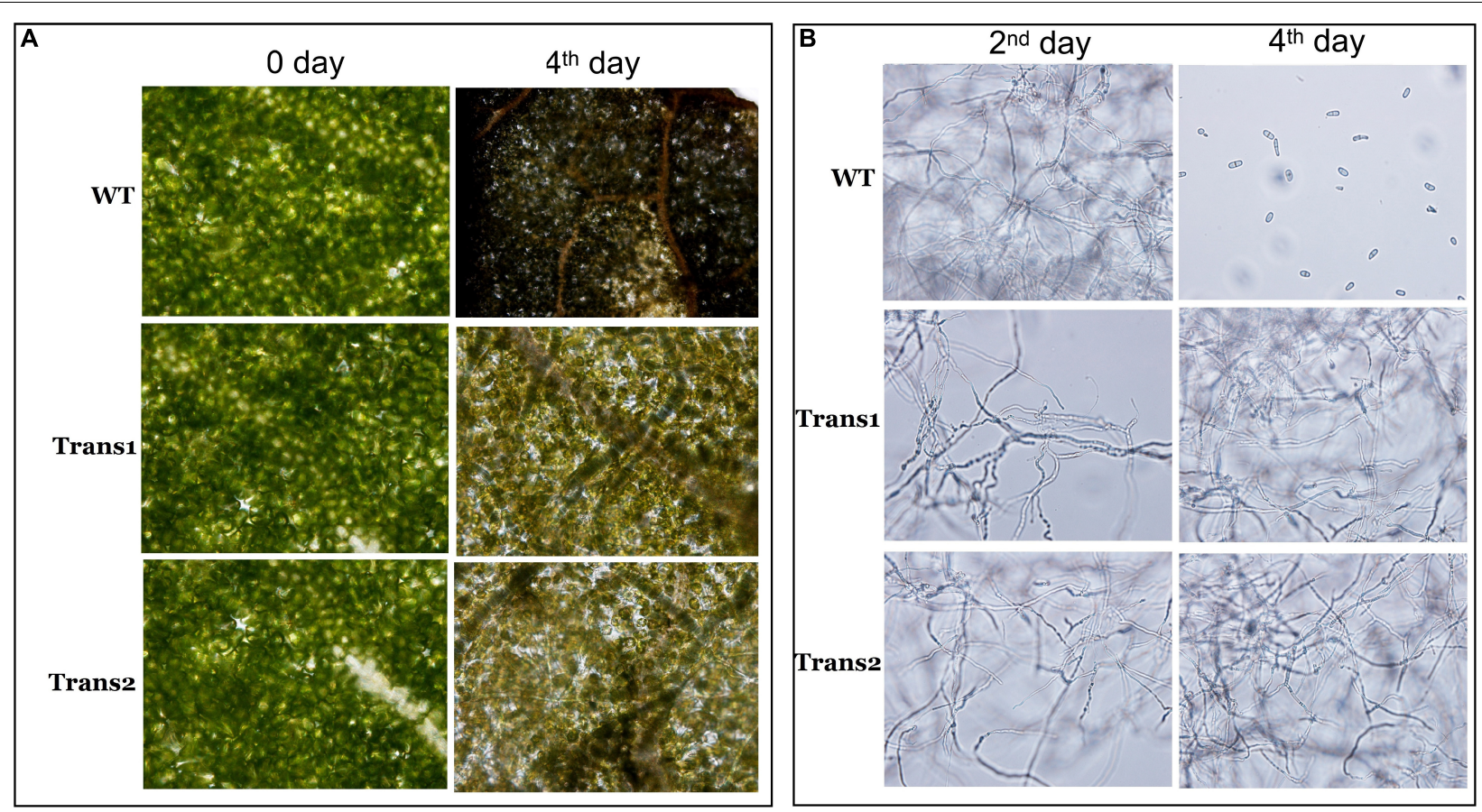

FIGURE 7 | Effects of inoculation with S. populiperda on disease leaf symptoms. (A) Microscopic observation of changes in transgenic and WT leaf internal structure under inoculation with S. populiperda. (B) Microscopic observation of S. populiperda generation time under inoculation with S. populiperda. Three independent experiments were performed.

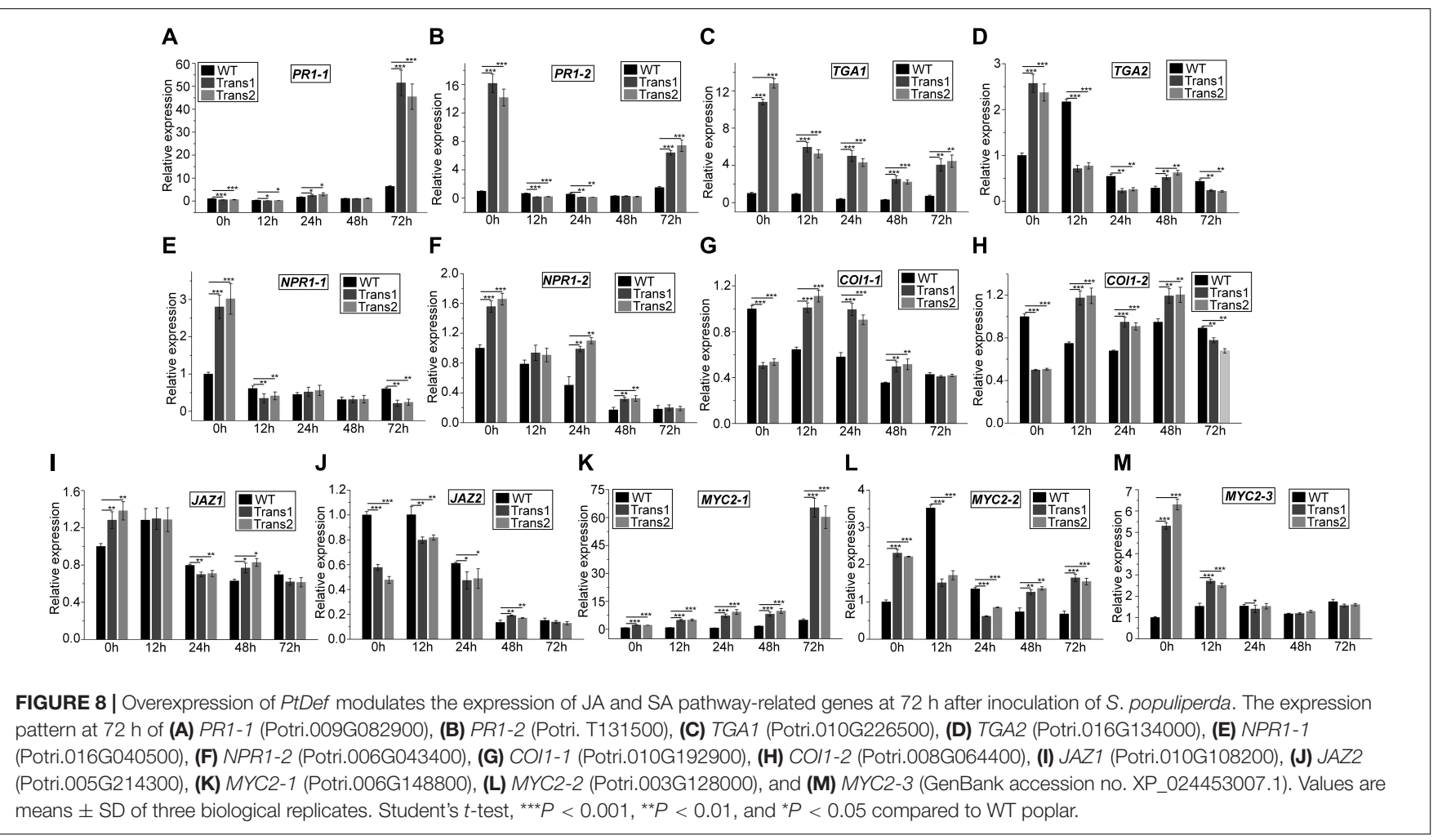


poplar than in WT at 0-72 h (Figure 8K). MYC2-2 expression was higher in WT than in transgenic poplar at $12-24 \mathrm{~h}$ (Figure 8L). MYC2-3 expression was highest in WT poplar at 0-12 h (Figure 8M).

Inoculation with $S$. populiperda modulated the expression of 13 genes related to SA and JA signal transduction in the transgenic and WT poplars. The expression of PR1-1 (12$72 \mathrm{~h}$ ), NPR1-2, TGA1, and MYC2-1 in transgenic poplar was higher than that in WT poplar. The expression of TGA1, TGA2, NPR1-1, and NPR1-2 was downregulated post-inoculation with S. populiperda in both transgenic and WT poplars, and the magnitude of downregulation was greater in the former.

\section{Effect of S. populiperda on the Expression of ROS-Related Genes and $\mathrm{H}_{2} \mathrm{O}_{2}$ Content}

$\mathrm{H}_{2} \mathrm{O}_{2}$ exerts a toxic effect on pathogens, participates in lignification, strengthens the cell walls of plants, hinders the penetration of fungi, induces programmed cell death, and acts as a signaling molecule (Heller and Tudzynski, 2011; Lehmann et al., 2015). The $\mathrm{H}_{2} \mathrm{O}_{2}$ content of the transgenic polar was higher than that of WT poplar at $48 \mathrm{~h}$ post-inoculation with $S$. populiperda. The $\mathrm{H}_{2} \mathrm{O}_{2}$ content of transgenic and WT poplars increased from 0 to $24 \mathrm{~h}$ post-inoculation, and then decreased significantly (Figure 9F). Therefore, the greater resistance to $S$. populiperda of transgenic than WT poplar may be related to their higher $\mathrm{H}_{2} \mathrm{O}_{2}$ content.

qRT-PCR showed that infection with $S$. populiperda downregulated APX and CAT expression in transgenic and WT poplar at most time points, and $A P X$ and $C A T$ expression was lower in transgenic than in WT poplar (Figures 9A,B). Additionally, RbohB expression in transgenic and WT poplar was upregulated at most time points and was markedly higher in transgenic poplar than in WT poplar (Figure 9C). Therefore, compared with WT poplar, transgenic poplar had lower $\mathrm{H}_{2} \mathrm{O}_{2}$ scavenging activity and higher production of $\mathrm{H}_{2} \mathrm{O}_{2}$ (Figure 9D). A high $\mathrm{H}_{2} \mathrm{O}_{2}$ level at the early stage $(0-48 \mathrm{~h})$ of infection may induce the expression of defense-related genes or function as a virulence factor (Apel and Hirt, 2004).

Although $\mathrm{H}_{2} \mathrm{O}_{2}$ is important for plant defense responses, excess $\mathrm{H}_{2} \mathrm{O}_{2}$ may contribute to fungal infection. SOD expression in transgenic poplar was markedly higher than that in WT poplar post-inoculation with $S$. populiperda (Figure 9E). Additionally, RbohA expression in transgenic poplar was higher than that in WT poplar except at $24 \mathrm{~h}$ (Figure 9F). The high SOD expression in transgenic poplar likely serves to prevent excessive accumulation of $\mathrm{H}_{2} \mathrm{O}_{2}$ in leaves. Therefore, the $\mathrm{H}_{2} \mathrm{O}_{2}$ content in transgenic poplar is maintained at a high level, which promotes resistance to $S$. populiperda but not to fungal infection.

\section{DISCUSSION}

Growing plants are vulnerable to pathogens and prone to a variety of diseases, which threaten crop yields, the environment, and sustainable agricultural development. Defensins are cationic antimicrobial peptides (AMPs) found in animals and plants and have broad-spectrum bactericidal activity (Thery and Arendt, 2018; Velivelli et al., 2018). In this study, we cloned a defensin gene from $P$. trichocarpa. Bioinformatics analysis revealed that PtDef had an ORF of 225 bp and encoded a 74 amino acid
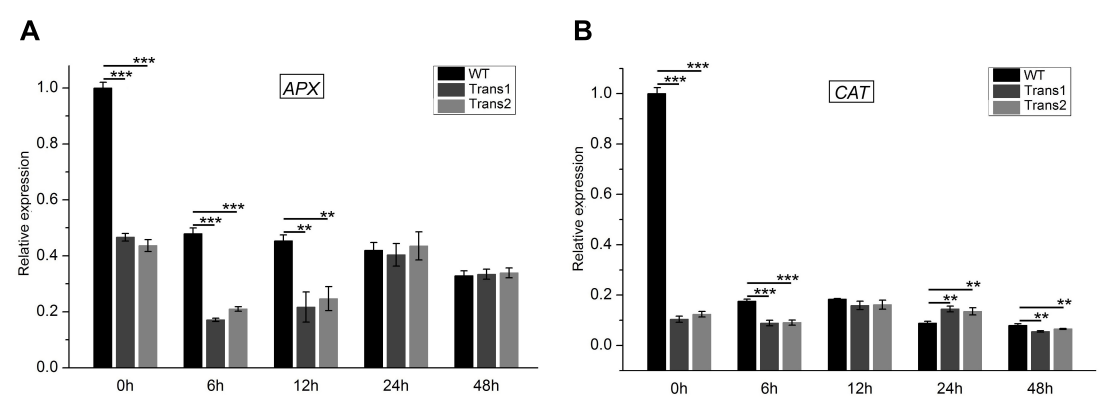

c
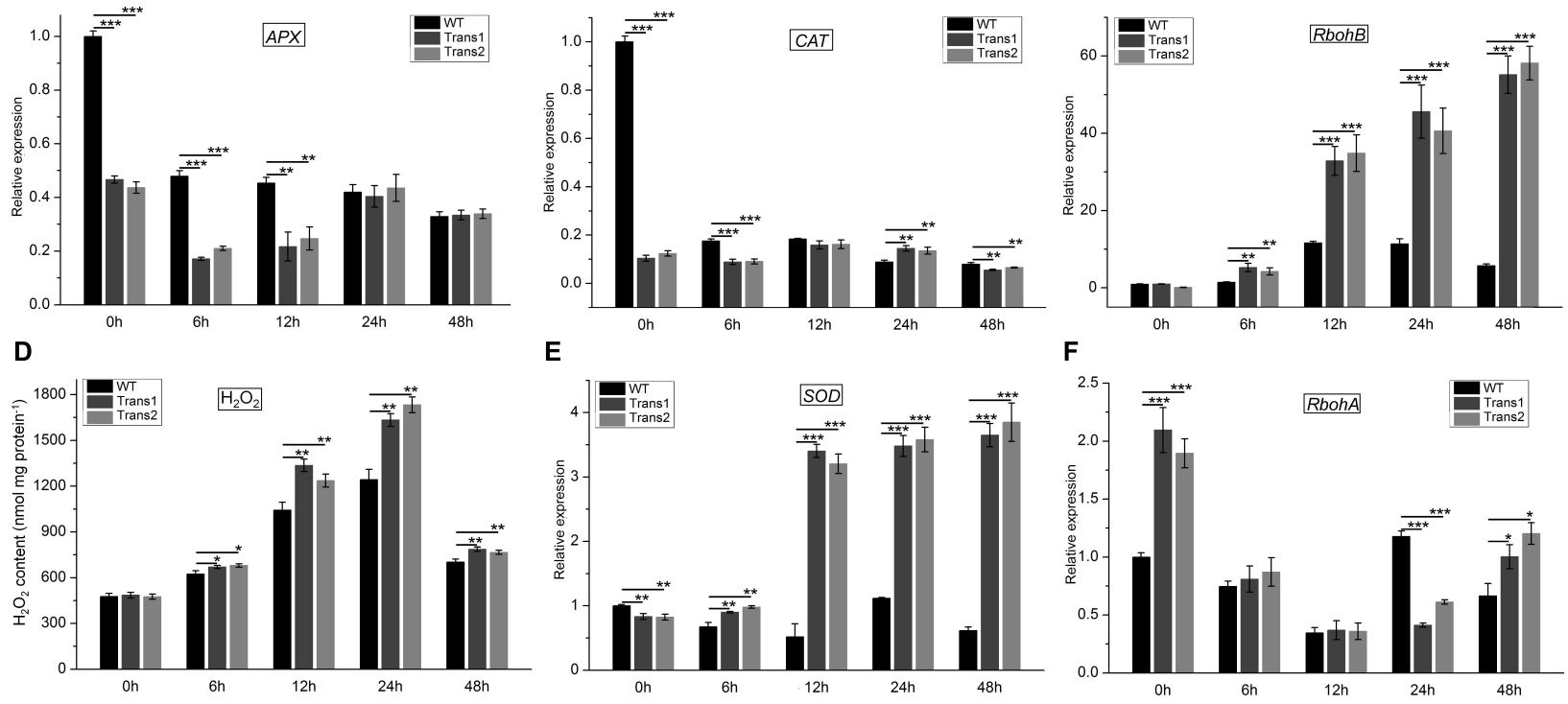

FIGURE $9 \mid \mathrm{H}_{2} \mathrm{O}_{2}$ content and expression of genes related to redox metabolism in transgenic and WT poplar leaves pre- and post-inoculation with S. populiperda. Expression of (A) APX, (B) CAT, (C) RbohB in transgenic and WT poplar post-inoculation with S. populiperda. (D) $\mathrm{H}_{2} \mathrm{O}_{2}$ content of transgenic and WT poplar following inoculation with S. populiperda. Expression of (E) SOD and (F) RbohA in transgenic and WT poplar following inoculation with $S$. populiperda. Values are means $\pm \mathrm{SD}$ of three biological replicates. Student's $t$-test, ${ }^{\star \star \star} P<0.001$, ${ }^{\star \star} P<0.01$, and ${ }^{\star} P<0.05$ compared to WT poplar. 
polypeptide. The N-terminus of PtDef had a signal peptide of 27 amino acids and eight cysteine residues. PtDef has a typical defensin structure with four disulfide bonds composed of eight cysteine residues (García-Olmedo et al., 1998). The defensin of Nigella Sativa has a strong inhibitory effect on Fusarium wilt, Botrytis cinerea, Fusarium graminearum, and other fungi (Rogozhin et al., 2011). Tf-gd1, a Trigonella foenum-graecum defensin, inhibits the growth of Thanatephorus cucumeris in vitro (Olli and Kirti, 2006), whereas the defensin of Brassica napus has bacteriostatic activity against Pyricularia oryzae and Xanthomonas oryzae (Motoshige et al., 2003). Agar diffusion assays revealed that PtDef is active against A. niger, A. Nees, M. corymbifer, M. populi, Rhizopus sp., and N. crassa. The expression of some plant defensin genes is constitutive and that of others is induced (Mirouze et al., 2006). The expression of the same defensin gene may also differ among varieties of the same species. For example, alfalfa defensin genes are expressed in Medicago sativa leaves, flowers, and seeds, but not in roots, while in Medicago truncatula, they are expressed in seeds but not in other organs (Hanks et al., 2005). In this study, PtDef was expressed in petioles, roots, stems, and leaves of poplar leaves, and was highest in petioles, followed by roots, stems, and leaves. qRT-PCR analysis revealed that PtDef responded to a variety of stresses. We speculate that PtDef is involved in a large number of physiological processes.

The addition of IPTG to cultures reduced the growth rate of E. coli BL21 (DE3). Transcriptome analysis of recombinant E. coli BL21 (DE3) before (control) and after induction by IPTG revealed alterations in the expression levels of genes related to the TCA cycle, oxidative phosphorylation, RNA degradation, ribosome, DNA replication, glycolysis/gluconeogenesis, and pyruvate metabolism. Based on antibiotic-induced bacterial autolysis, we speculate that PtDef induces autolysis of $E$. coli. Bacterial resistance to traditional antibiotics will render treatment of infection increasingly problematic (Hamilton et al., 2009). Therefore, new antibiotics are urgently needed. AMPs have broad-spectrum antimicrobial activity, immune-regulatory functions, and are important in maintaining homeostasis (Bastos et al., 2018). Therefore, AMPs may be feasible as alternatives to antibiotics. However, some bacteria are resistant to the killing effect of AMPs (Frick et al., 2003), whether they are from the host or administered for therapeutic purposes. Additionally, we found that PtDef modulates the expression of TCS-related genes in E. coli. Because this system often participates in bacterial adaptation to adverse environments, we preliminarily speculate that E. coli activates TCS to promote such adaptation.

An improved understanding of the biological characteristics and functions of defensins has enabled progress in the application of defensin-encoding genes. Plant varieties with enhanced disease resistance can be produced by transforming exogenous diseaseresistance genes into the plant genome. Transfer of $B j D$ from mustard into tobacco and peanut resulted in transgenic tobacco resistant to Phytophthora infestans and Fusarium moniliforme and transgenic peanut resistant to late peanut leaf spot (Anuradha et al., 2008). Rice transformed with the DmAMPI gene of Dahlia merckii were resistant to infection by Magnaporthe oryzae and Rhizoctonia solani (Jha et al., 2009). In this study, we used the Agrobacterium infection method to transfer PtDef into Nanlin895 poplar (Populus $\times$ euramericana cv. Nanlin895). We assessed the rate of lesion development on leaves, degree of damage to leaves, and the generation time of $S$. populiperda. We found that transgenic poplar exhibited strong resistance to S. populiperda.

The plant disease resistance and defense response signaling pathways are termed the SA, ET, and JA pathways; these pathways are interconnected (Koornneef et al., 2008; Islam et al., 2019). They differ in the types of exogenous signals, special signal transduction regulators, types of activated effector genes, and groups of resistant pathogens. However, there are some crosslinks among these signaling pathways (Mur et al., 2006; Yasuda et al., 2008). Post-inoculation with S. populiperda, TGA1 and NPR1-2 expression in transgenic poplar was significantly higher than that in WT poplar. PR1-1 expression at 12, 24, and $72 \mathrm{~h}$ in transgenic poplar increased significantly and was significantly higher than that in WT poplar. The regulatory protein NPR1 is a key cross-point in signal transduction networks composed of various disease-resistance-signal-transduction pathways. NPR1 acts downstream of SA and interacts with TGA-like transcription factors that bind to the SA response elements in the PR1 promoter, regulating its expression (Kinkema et al., 2000; Chern et al., 2001; Fan and Dong, 2002). The disease-related protein PR1 triggers production of SAR, inducing broad-spectrum disease resistance (Balint-Kurti, 2019). NPR1-1, NPR1-2, TGA1, and TGA2 expression in transgenic poplar was significantly higher than that in WT poplar before infection, indicating greater SA accumulation pre-inoculation with $S$. populiperda. TGA, NPR1, and PR1 are signal molecule related genes that open the SA signal transduction pathway, causing SAR to be more strongly induced in transgenic poplar. This may explain the strong disease resistance of transgenic poplar.

The expression of MYC2-1, MYC2-2, and MYC2-3 was high in transgenic poplar pre-inoculation with $S$. populiperda. MYC2 and other transcription factors are produced in large quantities in plants at certain developmental stages or under conditions of stress. MYC2 upregulates the expression of the injury- and JA-activated genes VSP and LOX in a COI1-dependent manner, initiating the transcription of resistance-related genes (Cheong et al., 2002). Therefore, the high expression of MYC2-1, MYC22, and MYC2-3 in transgenic poplar at the early stage of stress may trigger the transcription of JA-related genes, thus enhancing disease resistance. The SA and JA signaling pathways exert both antagonistic and synergistic effects. JA inhibits the expression of PR1, a marker gene involved in the SA signaling pathway (Pieterse et al., 2009). However, Zehnder et al. (2001) observed that JA-mediated ISR induced by Erwinia tracheiphila did not affect the expression of $P R 1$ in the field. In the present study, expression of PR1-1 in transgenic poplar was lower than that in WT poplar, and PR1-2 expression in transgenic poplar was higher than that in WT poplar before $S$. populiperda infection. However, $P R 1-1$ and PR1-2 expression in transgenic poplar at $12 \mathrm{~h}$ was lower than that in WT poplar, and vice versa at $72 \mathrm{~h}$. Therefore, PR1-1 expression was downregulated before infection but was upregulated at 24 and 72 h. PR1-2 expression also increased at $72 \mathrm{~h}$. Coronatine-insensitive 1 (COI1) is a regulatory 
signal switch in the JA signaling pathway and is involved in JA metabolism (Feys and Parker, 2000; Shen et al., 2018). In the present study, COI1-1 and COI1-2 expression in transgenic poplar was lower than that in WT poplar before infection. COI11 and COI1-2 expression in the transgenic poplar was higher than that in the WT poplar at $12-48 \mathrm{~h}$. This may be due to the accumulation of more SA signaling molecules in transgenic poplar, which inhibited COI1 expression. The strong resistance of transgenic poplars to $S$. populiperda may be due to the upregulation of PR1-1 and MYC2-1 and downregulation of JAZ1, COI1-1, and COI1-2, leading to activation of the SA and JA signaling pathways. The signaling pathways that control poplar disease resistance warrant further investigation.

The rapid generation of ROS is termed an oxidative burst and is an early response of plants to pathogens. ROS include superoxide, hydrogen peroxide, and singlet oxygen, and are produced by plants under stresses such as cold, drought, salt, and pathogen invasion. Rapid generation of ROS is an important defense response of plants to pathogens (Torres et al., 2006). ROS play an important role in plant defense: upon pathogen invasion of resistant plants, the production of oxygen-decomposing enzymes such as APX and CAT is inhibited (Klessig et al., 2000), increasing ROS production and inducing an HR reaction (Melillo et al., 2011). In the present study, $A P X$ and CAT expression in transgenic poplar was significantly lower than that in WT poplar at most time points. Additionally, the $\mathrm{H}_{2} \mathrm{O}_{2}$ content of transgenic poplar was significantly higher than that of WT poplar, and the generation time of $S$. populiperda on the leaves of transgenic poplar was significantly longer than that on the leaves of WT poplar, suggesting that the transgenic poplar responded to $S$. populiperda by increasing $\mathrm{H}_{2} \mathrm{O}_{2}$ production. The production of ROS in plants induces allergic reactions, in some cases leading to cell death (Fonseca and Mysore, 2019). In plants, excess $\mathrm{H}_{2} \mathrm{O}_{2}$ can cause cell death (Siddique et al., 2014). SOD, CAT, and GST are key enzymes in plant oxygen metabolism, which eliminates excess ROS produced in response to pathogens (Foyer and Shigeoka, 2011). In the present study, SOD expression was higher in transgenic than in WT poplar, suggesting higher levels of antioxidants. Based on these findings, we speculate that the ROS content of transgenic poplars must be maintained at a certain level to resist pathogen invasion and prevent damage to the plant.

In conclusion, we cloned a defensin gene from $P$. trichocarpa and named it PtDef (GenBank accession number XP_002325735.1). Its ORF was 225 bp and encoded a 74 amino acid protein. Phylogenetic analysis revealed that PtDef is closely related to RcDef of $R$. communis (XP_025012078.1). Also, PtDef was expressed in all tissues of poplar, and its expression was induced by exogenous biotic and abiotic stresses. Additionally, PtDef inhibited the growth of several fungi. To investigate the mechanism by which PtDef inhibits E. coli BL21 (DE3), we performed transcriptome analysis; the results indicated that PtDef induced E. coli BL21 (DE3) autolysis. Overexpression of PtDef in Nanlin895 poplar enhanced its resistance to $S$. populiperda. We also explored the reasons for the pathogen resistance of transgenic poplar. The expression of genes related to JA and SA differed between transgenic and WT poplar infected with S. populiperda. Differences in the expression of genes related to SA and JA signaling may modulate the activity of those pathways. Further research is needed to investigate the control of the signaling pathways involved in the disease resistance of poplar.

\section{MATERIALS AND METHODS}

\section{Plant Culture and Treatments}

We cultured $P$. trichocarpa and Nanlin 895 under long-day conditions (16-h light/8-h dark) in 1/2 Murashige and Skoog (MS) medium ( $\mathrm{pH} 5.8$ ) at $23^{\circ} \mathrm{C}$ and $74 \%$ humidity. We extracted RNA of young and mature leaves, upper and lower stems, petioles, and roots using a Plant RNA Extraction Kit (Biomiga, Inc.). Leaves of WT poplar seedlings grown for 3 months were treated with $200 \mathrm{mM} \mathrm{NaCl}, 200 \mu \mathrm{M}$ ABA, $200 \mu \mathrm{M} \mathrm{SA}, 200 \mu \mathrm{M}$ $\mathrm{JA}$, or $2 \mathrm{mM} \mathrm{H}_{2} \mathrm{O}_{2}$ and sampled at $0,1,3,6,8,12,24$, and $48 \mathrm{~h}$. WT poplar seedlings were treated with Macrogol $6000\left(\mathrm{PEG}_{6000}\right)$ and at $4^{\circ} \mathrm{C}$ (cold stress), and sampled at $0,1,2,3,4,5,6$, and 7 days. We wounded poplar leaves at $23^{\circ} \mathrm{C}$ and samples were taken at $0,6,12,24,48$, and $72 \mathrm{~h}$. Leaves of poplar seedlings were inoculated with $S$. populiperda on potato dextrose agar (PDA) (Zhu et al., 2019) and sampled at 0, 6, 12, 24, 48, and $72 \mathrm{~h}$; a blank PDA plate was used as control. RNA was then extracted from the leaves using a Plant RNA Extraction Kit (Biomiga, Inc.).

\section{Gene Cloning and Sequence Analysis}

First-strand cDNA was synthesized using a Reverse Transcription Kit (TaKaRa, Japan) in reactions comprising 200 ng RNA template, $1 \mu \mathrm{l}$ enzyme mix 1, $1 \mu \mathrm{l}$ random 6-mers, $1 \mu \mathrm{l}$ OligodT primer, $4 \mu \mathrm{l} 5 \times$ PrimeScript buffer, and RNase-free $\mathrm{ddH}_{2} \mathrm{O}$ to a volume of $20 \mu \mathrm{l}$. PCR was performed at $42^{\circ} \mathrm{C}$ for $30 \mathrm{~min}$ and $85^{\circ} \mathrm{C}$ for $5 \mathrm{~s}$, yielding $20 \mu \mathrm{l} \mathrm{cDNA}$.

The National Center for Biotechnology Information (NCBI) database $^{1}$ was used to search for defensin-encoding genes in the $P$. trichocarpa genome and to design primers (Supplementary Table S1). Each reaction comprised $2 \mu \mathrm{l} \mathrm{cDNA} \mathrm{(template),} 2 \mu \mathrm{l}$ of the forward and reverse primers $(10 \mu \mathrm{M}), 0.5 \mu \mathrm{lraq}, 4 \mu \mathrm{l}$ dNTPs $(2.5 \mathrm{mM}), 5 \mu \mathrm{l} 10 \times \mathrm{PCR}$ buffer $\left(\mathrm{Mg}^{2+}\right)$, and sterile water to a volume of $50 \mu \mathrm{l}$. PCR was performed as follows: $95^{\circ} \mathrm{C}$ for $10 \mathrm{~min}$, followed by 35 cycles of $95^{\circ} \mathrm{C}$ for $0.5 \mathrm{~min}, 58^{\circ} \mathrm{C}$ for $0.5 \mathrm{~min}$, $72^{\circ} \mathrm{C}$ for $0.5 \mathrm{~min}$, and $72^{\circ} \mathrm{C}$ for $10 \mathrm{~min}$. PCR products were identified by electrophoresis in $1 \%$ agarose gel and purified using a commercial kit (Axygen, Sunzhou, China). The PCR product was ligated into the PEASY-T3 vector (TransGen Biotech, Beijing, China) and transformed into competent cells of E. coli TransT1 (TransGen Biotech). Positive clones were screened for sequencing by Nanjing Genscript Co. (Nanjing, China).

Protparam $^{2}$ was used to analyze the physicochemical properties of the amino acids of PtDef, and the Signal server ${ }^{3}$ was used for signal peptide analysis. A homology search for defensins was performed in the NCBI database, and a phylogenetic tree was constructed using the MEGA 5.0 software.

\footnotetext{
${ }^{1}$ http://www.ncbi.nlm.nih.gov/gorf/gorf.html

${ }^{2}$ http://web.expasy.org/protparam

${ }^{3}$ http://www.cbs.dtu.dk/services/SignalP/
} 


\section{Analysis of PtDef Expression}

We designed qRT-PCR primers specific for PtDef (Supplementary Table S1) using the Primer 3.0 software. PtActin (GenBank accession no. XM006370951.1) (Supplementary Table S1) was used as an internal reference, and qRT-PCR was performed using a StepOnePlus real-time fluorescence qPCR instrument (Applied Biosystems, Foster City, CA, United States). The qRT-PCR reactions comprised $10 \mu \mathrm{l}$ SYBR Green, $1 \mu \mathrm{l}$ forward primer $(10 \mu \mathrm{mol}), 1 \mu \mathrm{l}$ reverse primer $(10 \mu \mathrm{mol}), 1 \mu \mathrm{l}$ cDNA (template), and sterile water to a volume of $20 \mu \mathrm{l}$. qRTPCR involved 40 cycles of pre-denaturation at $95^{\circ} \mathrm{C}$ for $10 \mathrm{~min}$, denaturation at $95^{\circ} \mathrm{C}$ for $10 \mathrm{~s}$, and annealing at $60^{\circ} \mathrm{C}$ for $30 \mathrm{~s}$.

\section{Construction of PET-32a-PtDef and Recombinant Plasmid Expression}

Primers (Supplementary Table S1) were designed and synthesized based on the BamHI and NotI restriction sites in the PET-32a plasmid. The restriction enzymes BamHI and NotI (TaKaRa) were used to digest the PCR product and PET-32a plasmid, respectively, followed by ligation using $\mathrm{T} 4$ ligase (TaKaRa). Positive clones were electrophoresed in $1 \%$ agarose gel and sequenced.

The plasmid pET32a-PtDef was transformed into E. coli BL21 (DE3) (Solarbio, China). A single colony was selected on a plate containing $50 \mu \mathrm{g} / \mathrm{ml}$ ampicillin and cultured in liquid medium containing $50 \mu \mathrm{g} / \mathrm{ml}$ ampicillin. The expression of pET32a-PtDef in $E$. coli BL21 (DE3) was induced by $1 \mathrm{mmol} / \mathrm{L}$ isopropyl- $\beta-\mathrm{D}$ 1-thiogalactopyranoside (IPTG) in $100 \mathrm{ml}$ Luria-Bertani (LB) medium for $4 \mathrm{~h}$ at $30^{\circ} \mathrm{C}$. Cells were harvested by centrifugation at $5000 \mathrm{rpm}$ for $15 \mathrm{~min}$, resuspended in $10 \mathrm{ml}$ binding buffer (20 mM Tris-base, $500 \mathrm{mM} \mathrm{NaCl}$, and $20 \mathrm{mM}$ imidazole), frozen at $-20^{\circ} \mathrm{C}$, and thawed in an ice bath; lysozyme was then added for a final volume of $1 \mathrm{mg} / \mathrm{ml} \mathrm{Next}$, the mixture was placed on ice for $30 \mathrm{~min}$, Triton X-100 was added, and the concentration of the solution was diluted to $0.1 \%$. DNase and RNase $(5 \mu \mathrm{g} / \mathrm{ml})$ were added and the mixture was incubated at $4^{\circ} \mathrm{C}$ for $10 \mathrm{~min}$. Finally, DL-dithiothreitol (DTT) was added to a concentration of $1 \mathrm{mmol} / \mathrm{L}$, the mixture was centrifuged, and the supernatant was decanted and stored at $4^{\circ} \mathrm{C}$.

His-PtDef was purified by His-label affinity chromatography at a flow rate of $0.5 \mathrm{ml} / \mathrm{min}$ and a pressure of $0.25 \mathrm{MPa}$; the UV280 signal was set to zero. Samples were added after the baseline had stabilized. Next, wash buffer ( $20 \mathrm{mM}$ Tris-base, $500 \mathrm{mM} \mathrm{NaCl}$, and $50 \mathrm{mM}$ imidazole) was added until the baseline returned to zero and elution buffer ( $20 \mathrm{mM}$ Tris-base, $500 \mathrm{mM} \mathrm{NaCl}$, and $250 \mathrm{mM}$ imidazole) was applied to elute the target protein. The fractions recovered were evaluated by $12 \%$ SDS-PAGE.

\section{Antifungal Activity Assay}

Aspergillus niger, Alternaria Nees, Mucor corymbifer, Marssonina populi, Rhizopus sp., and Neurospora crassa were inoculated on PDA and activated in a $25^{\circ} \mathrm{C}$ incubator. The fungi were resuspended in $0.9 \% \mathrm{NaCl}(\mathrm{pH} 7.0)$ and transferred to liquid PDA, which was poured into Petri dishes. After setting, holes were made in the PDA. We dissolved $120 \mu \mathrm{ltDef}$ in $0.9 \% \mathrm{NaCl}$ (pH 7.0) to $40,20,10,5,2.5,1.25$, and $0.625 \mu \mathrm{g} / \mathrm{ml}$ and added the solutions to PDA in triplicate. PDA containing $0.9 \% \mathrm{NaCl}(\mathrm{pH}$ 7.0) was used as a negative control. The plates were incubated at $25^{\circ} \mathrm{C}$ for $72 \mathrm{~h}$ until observation of mycelia.

\section{Growth of E. coli BL21 (DE3)}

Recombinant E. coli BL21 (DE3) was activated on LB agar containing $50 \mu \mathrm{g} / \mathrm{ml}$ ampicillin. Monoclones were cultured in LB containing $50 \mu \mathrm{g} / \mathrm{ml}$ ampicillin overnight at $37^{\circ} \mathrm{C}$ with shaking at $220 \mathrm{rpm}$. The following day, recombinant E. coli BL21 (DE3) was transferred to fresh LB at a 1:100 dilution and cultured to an $\mathrm{OD}_{600}$ of 0.4 , after which IPTG was added to $1 \mathrm{mM}$. $\mathrm{OD}_{600}$ was then evaluated at 30 -min intervals. A culture not induced by IPTG was used as the control.

\section{Transcriptome Analysis of E. coli BL21 (DE3)}

We performed transcriptome analysis of recombinant $E$. coli BL21 (DE3) before (control) and after PtDef induction by IPTG. RNA was extracted from E. coli BL21 (DE3) using an RNA Extraction Kit (Biomiga, Inc.) its integrity was investigated by electrophoresis in $2 \%$ agarose gel. RNA concentration and purity were determined using Nanodrop and Qubit instruments, respectively. The E. coli transcriptome was sequenced by Allwegene Bioinformatics Technology Co., Ltd. (Beijing, China). Data filtering and quality evaluation were performed, and the transcripts of clean reads were spliced. We used the $\mathrm{Nr}$, Pfam, eggNOG, Swiss-prot, Kyoto Encyclopedia of Genes and Genomes (KEGG), and Gene Ontology (GO) databases for subsequent analyses.

\section{Construction and Transformation of an Overexpression Plasmid}

Forward and reverse primers (Supplementary Table S1) were designed according to the gateway entry vector $\mathrm{pENTR/D-}$ TOPO (Invitrogen, Carlsbad, CA, United States). Following amplification by PCR, the BP reaction was performed to construct the plasmid pENTR-PtDef by homologous recombination. Next, the recombinant plasmid pGWB9-PtDef (pGWB9 accession no. AB289772) was produced by LR reaction based on LR Clonase II and transformed into A. tumefaciens EHA105 (Solarbio, China).

Nanlin895 poplar (Populus $\times$ euramericana cv. Nanlin895) transformation was performed as follows. Nanlin895 poplar leaves and petioles were precultured for 3 days on differentiation MS medium ( $\mathrm{pH}$ 5.8). A. tumefaciens EHA105 harboring pGWB9-PtDef was incubated at $28^{\circ} \mathrm{C}$ to an $\mathrm{OD}$ of 0.8 , harvested by centrifugation at $5000 \mathrm{rpm}$ for $15 \mathrm{~min}$, resuspended in liquid sucrose-free MS medium, and used to infect Nanlin895 poplar leaves and petioles. Finally, Nanlin895 poplar leaves and petioles were transferred to differentiation MS medium and resistant plants were obtained by screening on bud elongation MS medium and screening rooting MS medium (1/2 MS medium) (Movahedi et al., 2015, 2018).

Total DNA of WT and transgenic poplars was extracted using a DNA Extraction Kit (Biomiga, Inc.) and amplified by PCR using PtDef-F and PtDef-R, and 35S (Supplementary Table S1) 
and PtDef-R, as forward and reverse primers, respectively. Total RNA of WT and transgenic poplars was extracted using an RNA Extraction Kit (Biomiga, Inc.) transcribed into cDNA, and subjected to qRT-PCR as described above.

\section{Analysis of Septotinia populiperda Resistance}

Septotinia populiperda was cultured on PDA at $25^{\circ} \mathrm{C}$ for 7 days. Leaves were moisturized using moist sterile degreased cotton. Holes were made in leaves of 4-month-old WT and transgenic poplars using a hole punch and inoculated with $S$. populiperda. Leaves were cultured for $24 \mathrm{~h}$ in the dark at 95\% humidity and $23^{\circ} \mathrm{C}$, and transferred to normal conditions (16 h light $/ 8 \mathrm{~h}$ dark, $23^{\circ} \mathrm{C}, 74 \%$ humidity). Infection was monitored daily; the expanded range of $S$. populiperda in leaves was measured, and the degree of leaf damage $S$. populiperda growth was observed by microscopy. Analysis of genes related to the JA and SA signaling pathways; APX, CAT, SOD, RbohA, and RbohB expression levels; and $\mathrm{H}_{2} \mathrm{O}_{2}$ content.

PR1, NPR1, and TGA (related to SA signal transduction) and $J A Z, C O I 1$, and MYC2 (related to JA signal transduction) were amplified by qRT-PCR using the primers listed in Supplementary Table S1. The effects of S. populiperda infection on the expression of 13 genes were analyzed by qRT-PCR. Expression of the genes encoding ROS-scavenging enzymes APX, CAT, and GST and ROS-producing enzymes $R b o h A$ and $R b o h B$ were analyzed by qRT-PCR as described above using the primers listed in Supplementary Table $\mathrm{S} 1$.

$\mathrm{H}_{2} \mathrm{O}_{2}$ content was determined as previously described (Gay et al., 1999). Leaf samples inoculated or not with $S$. populiperda were collected at $0,6,12,24$, and $48 \mathrm{~h}$ and placed in liquid nitrogen; $1.5 \mathrm{ml}$ of $500 \mathrm{mM}$ boric acid buffer ( $\mathrm{pH} 8.4$ ) was added. Samples were then mixed gently in an ice bath for $10 \mathrm{~min}$ and centrifuged at $10,000 \mathrm{rpm}$ for $20 \mathrm{~min}$ at $4^{\circ} \mathrm{C}$. Sediments were removed and supernatants were collected, and $1 \mathrm{ml}$ of a solution containing $25 \mathrm{mM}$ ferrous sulfate, $100 \mathrm{mM}$ sorbitol, $0.125 \mathrm{mM}$ xylenol orange, $25 \mathrm{mM}$ ammonium sulfate, and $100 \mathrm{mM}$ sulfuric acid was added. A standard curve was generated from $\mathrm{H}_{2} \mathrm{O}_{2}$ standards at $560 \mathrm{~nm}$ using a Genesys $10 \mathrm{~S}$ UV-vis spectrophotometer (Thermo Scientific, Waltham, MA, United States).

\section{DATA AVAILABILITY STATEMENT}

The raw RNA sequencing data were deposited in the NCBI Sequence Read Archive (SRA) with the accession number SRR9126592.

\section{AUTHOR CONTRIBUTIONS}

HW and AM designed the experiments. HW and AM wrote the manuscript. HW, AM, CX, WS, PW, and DL read and reviewed the manuscript. TY and QZ supervised this project. QZ funded this project.

\section{FUNDING}

This work was supported by the National Science Foundation of China (No. 31570650), and the Priority Academic Program Development of Jiangsu Higher Education Institutions.

\section{SUPPLEMENTARY MATERIAL}

The Supplementary Material for this article can be found online at: https://www.frontiersin.org/articles/10.3389/fmicb.2020. 00106/full\#supplementary-material

FIGURE S1 | Nucleotide and deduced amino acid sequences of PtDef (GenBank accession no. XP_002325735.1). The ATG (initiation codon) is boxed and the TAG (termination codon) is indicated by a five-pointed star.

FIGURE S2 | Amino acid sequence alignment of several plant defensins using ClustalW; red arrow, cysteine residues.

FIGURE S3 | Amino acid composition distribution of PtDef. Connections between cysteine residues represent disulfide bonds.

FIGURE S4 | Phylogenetic analysis of PtDef. The phylogenetic tree was constructed using the neighbor-joining method with MEGA 5.1 software and bootstrapped 1000 times. The GenBank accession numbers of the Def sequences are: Ricinus communis (XM_025156310.1), Juglans regia (XM_018955615.1), Morus notabilis (XM_024165652.1), Quercus suber (XM_024039378.1), Nicotiana tomentosiformis (XM_009592617.2), Cephalotus follicularis (BDDD01004928.1), Nicotiana sylvestris (XM_009781749.1), Nicotiana attenuata (XM_019404257.1), Durio zibethinus (XM_022879395.1), Theobroma cacao (XM_018119227.1), Ziziphus jujuba (XM_016024166.2), Manihot esculenta (XM_021763356.1), Medicago truncatula (XM_013606172.2), Trifolium subterraneum (DF973199.1), Olea europaea var. Sylvestris (XM_022990840.1), Arachis duranensis (XM_016100210.2), Cajanus cajan (XM_020358126.1), Nicotiana tabacum (XM_016595723.1), Chenopodium quinoa (XM_021877694.1), Momordica charantia (XM_022290632.1), Capsicum annuum (XM_016700063.1), Citrus sinensis (XM_025094762.1), Artemisia annua (PKPP01003814.1), and Arabidopsis thaliana (NP_201171.1).

FIGURE S5 | (A) Growth curve of E. coli BL (DE3) induced or not by IPTG (Student's $t$-test, ${ }^{* * *} P<0.001$ and ${ }^{* *} P<0.01$ ). (B) Mechanism by which antibiotics promote autolysis of bacteria.

FIGURE S6 | (A) Kyoto Encyclopedia of Genes and Genomes (KEGG) pathway annotation of differentially expressed genes (DEGs) related to the TCA cycle; $a c n A, y d b K$, frd $C$, frdD, frd $A$, and frdB were downregulated and fum $C$, sdhD, $s d h C$, gltA, sdh $B, s d h A$, and $s u c A$ were upregulated. (B) KEGG pathway annotation of DEGs related to oxidative phosphorylation; nuo $F, n u o E$, nuoC, ppa, $a p p C$, frdC, $a p p B$, frdD, frd $A$, and frdB were downregulated and $c y \circ A, c y o E$, $s d h D, s d h C, s d h B$, and $s d h A$ were upregulated.

FIGURE S7 | (A) KEGG pathway annotation of DEGs related to RNA degradation; groL, eno, and $r$ ne were downregulated and $r h I B$, $h f q$, rho, and $r p p H$ were upregulated. (B) KEGG pathway annotation of DEGs related to the ribosome; $r p / C, r p / D, r p / W, r p / B, r p s S, r p / N, r p s C, r p I P, r p s M, r p s K, r p / Y, r p / F, r p s D, r p s B$, $r p s J, r p / X, r p / M, r p s H, r p s N, r p s E, r p / R, r p / K, r p / A, r p s F, r p s G$, and $r p s L$ were downregulated. (C) KEGG pathway annotation of DEGs related to DNA replication; dnaE was downregulated and $r n h A$ was upregulated.

FIGURE S8 | (A) KEGG pathway annotation of DEGs related to glycolysis/gluconeogenesis; nuoF, nuoE, nuoC, ppa, appC, frdC, appB, frdD, frdA, and frdB were downregulated. (B) KEGG pathway annotation of DEGs related to pyruvate metabolism; poxB, NA, pykF, aldB, pykA, ydbK, frdC, frdD, $f r d A$, frdB, and $p f B$ were downregulated.

FIGURE S9 | KEGG pathway annotations of DEGs.

FIGURE S10 | Scatter plot of KEGG pathway enrichment. The abscissa is the enrichment factor of the pathway, the ordinate is the name of the pathway, the 
number of different genes in the pathway is indicated by the size of the point, and the size of the $P$-value is indicated by the color of the point (the smaller $P$-value, the closer the color to red).

FIGURE S11 | Molecular identification of regenerated PtDef transgenic plants. (A) Identification of PtDef in the genome of transgenic and WT poplar by PCR using the PtDef-F and PtDef-R forward and reverse primers, respectively. Lane M, molecular mass marker; Lane 1, negative control (genome of WT poplar as the template); Lanes 2-11, transgenic lines 1-10 (Trans1-Trans10). (B) Identification of PtDef in the genome of transgenic and WT poplar by PCR using the 35S promoter

\section{REFERENCES}

Almeida, M. S., Cabral, K. M., Kurtenbach, E., Almeida, F. C., and Valente, A. P. (2002). Solution structure of Pisum sativum defensin 1 by high resolution NMR: plant defensins, identical backbone with different mechanisms of action. J. Mol. Biol. 315, 749-757. doi: 10.1006/jmbi.2001.5252

Anuradha, T. S., Divya, K., Jami, S. K., and Kirti, P. B. (2008). Transgenic tobacco and peanut plants expressing a mustard defensin show resistance to fungal pathogens. Plant Cell Rep. 27, 1777-1786. doi: 10.1007/s00299-008-0596-8

Apel, K., and Hirt, H. (2004). Reactive oxygen species: metabolism, oxidative stress, and signal transduction. Annu. Rev. Plant Biol. 55, 373-399. doi: 10.1146/ annurev.arplant.55.031903.141701

Baharoglu, Z., Bikard, D., and Mazel, D. (2010). Conjugative DNA transfer induces the bacterial SOS response and promotes antibiotic resistance development through integron activation. PLoS Genet. 6:e1001165. doi: 10.1371/journal. pgen. 1001165

Balint-Kurti, P. (2019). The plant hypersensitive response: concepts, control and consequences. Mol. Plant Pathol. 20, 1163-1178.

Bastos, P., Trindade, F., da Costa, J., Ferreira, R., and Vitorino, R. (2018). Human antimicrobial peptides in bodily fluids: current knowledge and therapeutic perspectives in the postantibiotic era. Med. Res. Rev. 38, 101-146. doi: 10.1002/ med. 21435

Beceiro, A., Tomás, M., and Bou, G. (2013). Antimicrobial resistance and virulence: a successful or deleterious association in the bacterial world? Clin. Microbiol. Rev. 26, 185-230. doi: 10.1128/cmr.00059-12

Bloch, C., and Richardson, M. (1991). A new family of small (5 kDa) protein inhibitors of insect $\alpha$-amylases from seeds or sorghum (Sorghum bicolor (L) Moench) have sequence homologies with wheat $\gamma$-purothionins. FEBS Lett. 279, 101-104. doi: 10.1016/0014-5793(91)80261-Z

Brodelius, P., and Xue, Z. T. (1997). Isolation and characterization of a cDNA from cell suspension cultures of Vanilla planifolia Andr. Encoding 4-Coumarate: CoA Ligase. Plant Physiol. Biochem. 35, 497-506.

Broekaert, W. F., Terras, F. R., Cammue, B. P., and Osborn, R. W. (1995). Plant defensins: novel antimicrobial peptides as components of the host defense system. Plant Physiol. 108, 1353-1358. doi: 10.1104/pp.108.4.1353

Cheong, Y. H., Chang, H. S., Gupta, R., Wang, X., Zhu, T., and Luan, S. (2002). Transcriptional profiling reveals novel interactions between wounding, pathogen, abiotic stress, and hormonal responses in Arabidopsis. Plant Physiol. 129, 661-677. doi: 10.1104/pp.002857

Chern, M. S., Fitzgerald, H. A., Yadav, R. C., Canlas, P. E., Dong, X., and Ronald, P. C. (2001). Evidence for a disease-resistance pathway in rice similar to the NPR-mediated signaling pathway in Arabidopsis. Plant J. 27, 101-113. doi: 10.1046/j.1365-313x.2001.01070.x

Cools, T. L., Vriens, K., Struyfs, C., Verbandt, S., Ramada, M. H., Brand, G. D., et al. (2017). The antifungal plant defensin HsAFP1 is a phosphatidic acid-interacting peptide inducing membrane permeabilization. Front. Microbiol. 8:2295. doi: $10.3389 /$ fmicb.2017.02295

Cui, H. G. E., Kracher, B., Qiu, J., Bautor, J., and Parker, J. E. (2017). A core function of EDS1 with PAD4 is to protect the salicylic acid defense sector in Arabidopsis immunity. New Phytol. 213, 1802-1817. doi: 10.1111/nph.14302

Da-Hui, L., Gui-Liang, J., Ying-Tao, Z., and Tie-Min, A. (2007). Bacterial expression of a Trichosanthes kirilowii defensin (TDEF1) and its antifungal activity on Fusarium oxysporum. Appl. Microbiol. Biotechnol. 74, 146-151. doi: 10.1007/s00253-006-0631-z as the forward primer and PtDef-R as the reverse primer. Lane $\mathrm{M}$, molecular mass marker; Lane 1, negative control (WT poplar genome as the template); Lanes 2-11, transgenic lines 1-10 (Trans1-Trans10). (C) Analysis of PtDef expression in transgenic and WT poplar by real-time RT-PCR. Values are means \pm SD of three biological replicates. Student's $t$-test, ${ }^{* * *} P<0.001$ compared to WT poplar.

TABLE S1 | Primers used in this study.

TABLE S2 | Kyoto Encyclopedia of Genes and Genomes (KEGG) pathway annotation of differentially expressed genes (DEGs).

De Coninck, B., Timmermans, P., Vos, C., Cammue, B. P., and Kazan, K. (2015). What lies beneath: belowground defense strategies in plants. Trends Plant Sci. 20, 91-101. doi: 10.1016/j.tplants.2014.09.007

De Coninck, B., Verheesen, P., Vos, C. M., Van Daele, I., De Bolle, M. F., Vieira, J. V., et al. (2017). Fungal glucosylceramide-specific camelid single domain antibodies are characterized by broad spectrum antifungal activity. Front. Microbiol. 8:1059. doi: 10.3389/fmicb.2017.01059

Do, H. M., Lee, S. C., Jung, H. W., Sohn, K. H., and Hwang, B. K. (2004). Differential expression and in situ localization of a pepper defensin (CADEF1) gene in response to pathogen infection, abiotic elicitors and environmental stresses in Capsicum annuum. Plant Sci. 166, 1297-1305. doi: 10.1016/j.plantsci.2004. 01.008

Fan, W., and Dong, X. (2002). In vivo interaction between NPR1 and transcription factor TGA2 leads to salicylic acid-mediated gene activation in Arabidopsis. Plant Cell 14, 1377-1389. doi: 10.1105/tpc.001628

Feys, B. J., and Parker, J. E. (2000). Interplay of signaling pathways in plant disease resistance. Trends Genet. 16, 449-455. doi: 10.1016/s0168-9525(00) 02107-7

Fonseca, J. P., and Mysore, K. S. (2019). Genes involved in nonhost disease resistance as a key to engineer durable resistance in crops. Plant Sci. 279, 108-116. doi: 10.1016/j.plantsci.2018.07.002

Foyer, C. H., and Shigeoka, S. (2011). Understanding oxidative stress and antioxidant functions to enhance photosynthesis. Plant Physiol. 155, 93-100. doi: 10.1104/pp.110.166181

Frick, I. M., Åkesson, P., Rasmussen, M., Schmidtchen, A., and Björck, L. (2003). SIC, a secreted protein of Streptococcus pyogenes that inactivates antibacterial peptides. J. Biol. Chem. 278, 16561-16566. doi: 10.1074/jbc.m301995200

Fu, Z. Q., Yan, S., Saleh, A., Wang, W., Ruble, J., Oka, N., et al. (2012). NPR3 and NPR4 are receptors for the immune signal salicylic acid in plants. Nature 486, 228-232. doi: 10.1038/nature11162

Gabriel, D. W., and Rolfe, B. G. (1990). Working models of specific recognition in plant-microbe interactions. Ann. Rev. Phytopathol. 28, 365-391. doi: 10.1146/ annurev.py.28.090190.002053

Ganz, T. (2003). Defensins: antimicrobial peptides of innate immunity. Nat. Rev. Immunol. 3, 710-720. doi: 10.1038/nri1180

Gao, A. G., Hakimi, S. M., Mittanck, C. A., Wu, Y., and Rommens, C. M. T. (2001). Fungal pathogen protection in potato by expression of a plant defensin peptide. Nat. Biotechnol. 18, 1307-1310. doi: 10.1038/82436

García-Olmedo, F., Molina, A., Alamillo, J. M., and Rodríguez-Palenzuéla, P. (1998). Plant defense peptides. Peptide Sci. 47, 479-491.

Gay, C., Collins, J., and Gebicki, J. M. (1999). Hydroperoxide assay with the ferricxylenol orange complex. Anal Biochem. 273, 149-155. doi: 10.1006/abio.1999. 4208

Halitschke, R., Ziegler, J., Keinänen, M., and Baldwin, I. T. (2004). Silencing of hydroperoxide lyase and allene oxide synthase reveals substrate and defense signaling crosstalk in Nicotiana attenuata. Plant J. 40, 35-46. doi: 10.1111/j. 1365-313x.2004.02185.x

Hamilton, B. E., Martin, J. A., and Ventura, S. J. (2009). United States department of health and human services, centers for disease control and prevention. Natl. Center Health Stat. Natl. Vital Stat. Rep. 57:12.

Hanks, J. N., Snyder, A. K., Graham, M. A., Shah, R. K., Blaylock, L. A., Harrison, M. J., et al. (2005). Defensin gene family in Medicago truncatula: structure, expression and induction by signal molecules. Plant Mol. Biol. 58, 385-399. doi: $10.1007 /$ s11103-005-5567-7 
Heller, J., and Tudzynski, P. (2011). Reactive oxygen species in phytopathogenic fungi: signaling, development, and disease. Ann. Rev. Phytopathol. 49, 369-390. doi: 10.1146/annurev-phyto-072910-095355

Islam, W., Naveed, H., Zaynab, M., Huang, Z., and Chen, H. Y. (2019). Plant defense against virus diseases; growth hormones in highlights. Plant Signal. Behav. 14:1596719. doi: 10.1080/15592324.2019.1596719

Jha, S., Tank, H. G., Prasad, B. D., and Chattoo, B. B. (2009). Expression of DmAMP1 in rice confers resistance to Magnaporthe oryzae and Rhizoctonia solani. Transgen. Res. 18, 59-69. doi: 10.1007/s11248-008-9196-1

Kaul, M., Mohren, G. M. J., and Dadhwal, V. K. (2010). Carbon storage and sequestration potential of selected tree species in India. Mitigat. Adapt. Strat. Glob. Change 15, 489-510. doi: 10.1007/s11027-010-9230-5

Kinkema, M., Fan, W., and Dong, X. (2000). Nuclear localization of NPR1 is required for activation of PR gene expression. Plant Cell 12, 2339-2350. doi: 10.1105/tpc.12.12.2339

Klessig, D. F., Durner, J., Noad, R., Navarre, D. A., Wendehenne, D., Kumar, D., et al. (2000). Nitric oxide and salicylic acid signaling in plant defense. Proc. Natl. Acad. Sci. U.S.A. 97, 8849-8855.

Kohanski, M. A., Dwyer, D. J., Hayete, B., Lawrence, C. A., and Collins, J. J. (2007). A common mechanism of cellular death induced by bactericidal antibiotics. Cell 130, 797-810. doi: 10.1016/j.cell.2007.06.049

Koornneef, A., Leon-Reyes, A., Ritsema, T., Verhage, A., Den Otter, F. C., Van Loon, L. C., et al. (2008). Kinetics of salicylate-mediated suppression of jasmonate signaling reveal a role for redox modulation. Plant Physiol. 147, 1358-1368. doi: 10.1104/pp.108.121392

Lehmann, S., Serrano, M., L'Haridon, F., Tjamos, S. E., and Metraux, J. P. (2015). Reactive oxygen species and plant resistance to fungal pathogens. Phytochemistry 112, 54-62. doi: 10.1016/j.phytochem.2014.08.027

Melillo, M. T., Leonetti, P., Leone, A., Veronico, P., and Bleve-Zacheo, T. (2011). ROS and NO production in compatible and incompatible tomato-Meloidogyne incognita interactions. Eur. J. Plant Pathol. 130, 489-502. doi: 10.1007/s10658011-9768-4

Méndez, E., Rocher, A., Calero, M., Girbés, T., Citores, L., and Soriano, F. (1996). Primary structure of $\omega$-hordothionin, a member of a novel family of thionins from barley endosperm, and its inhibition of protein synthesis in eukaryotic and prokaryotic cell-free systems. Eur. J. Biochem. 239, 67-73. doi: 10.1111/j.14321033.1996.0067u.x

Mirouze, M., Sels, J., Richard, O., Czernic, P., Loubet, S., Jacquier, A., et al. (2006). A putative novel role for plant defensins: a defensin from the zinc hyperaccumulating plant. Arabidopsis halleri, confers zinc tolerance. Plant J. 47, 329-342. doi: 10.1111/j.1365-313x.2006.02788.x

Motoshige, K. T., Nakajima, T., Tsuyoshi, Y., Koichi, M., Tetsuo, O., Fukumoto, F., et al. (2003). Genetic engineering for disease resistance in rice (Oryza sativa 1.) using antimicrobial peptides. Jpn. Agric. Res. Q. 37, 71-76. doi: 10.6090/jarq. 37.71

Movahedi, A., Zhang, J., Gao, P., Yang, Y., Wang, L., Yin, T., et al. (2015). Expression of the chickpea CarNAC3 gene enhances salinity and drought tolerance in transgenic poplars. Plant Cell Tissue Organ Cult. 120, 141-154. doi: $10.1007 / \mathrm{s} 11240-014-0588-\mathrm{z}$

Movahedi, A., Zhang, J., Sun, W., Mohammadi, K., Yaghuti, A. A. Z., Wei, H., et al. (2018). Functional analyses of PtRDM1 gene overexpression in poplars and evaluation of its effect on DNA methylation and response to salt stress. Plant Physiol. Biochem. 127, 64-73. doi: 10.1016/j.plaphy.2018.03.011

Mur, L. A., Kenton, P., Atzorn, R., Miersch, O., and Wasternack, C. (2006). The outcomes of concentration-specific interactions between salicylate and jasmonate signaling include synergy, antagonism, and oxidative stress leading to cell death. Plant Physiol. 140, 249-262. doi: 10.1104/pp.105. 072348

Olli, S., and Kirti, P. B. (2006). Cloning, characterization and antifungal activity of defensin Tfgd1 from Trigonella foenum-graecum L. BMB Rep. 39, 278-283. doi: 10.5483/bmbrep.2006.39.3.278

Parisi, K., Shafee, T. M., Quimbar, P., van der Weerden, N. L., Bleackley, M. R., and Anderson, M. A. (2018). The evolution, function and mechanisms of action for plant defensins. Semin. Cell Dev. Biol. 88, 107-118. doi: 10.1016/j.semcdb.2018. 02.004

Pelegrini, P. B., Lay, F. T., Murad, A. M., Anderson, M. A., and Franco, O. L. (2008). Novel insights on the mechanism of action of $\alpha$-amylase inhibitors from the plant defensin family. Proteins 73, 719-729. doi: 10.1002/prot.22086
Pieterse, C. M., Leon-Reyes, A., Van der Ent, S., and Van Wees, S. C. (2009). Networking by small-molecule hormones in plant immunity. Nat. Chem. Biol. 5, 308-316. doi: 10.1038/nchembio.164

Pothana, A., Bhatnagar-Mathur, P., Yeshvekar, R. K., and Sharma, K. K. (2019). Plant defensins: tissue specific expression leading to distinctive functions. Curr. Trends Biotechnol. Pharm. 13, 212-231.

Rogozhin, E. A., Oshchepkova, Y. I., Odintsova, T. I., Khadeeva, N. V., Veshkurova, O. N., Egorov, T. A., et al. (2011). Novel antifungal defensins from Nigella sativa L. seeds. Plant Physiol. Biochem. 49, 131-137. doi: 10.1016/j.plaphy.2010. 10.008

Scheres, B., and van der Putten, W. H. (2017). The plant perceptron connects environment to development. Nature 543,337-345. doi: 10.1038/nature22010

Seifi, H. S., and Shelp, B. J. (2019). Spermine differentially refines plant defense responses against biotic and abiotic stresses. Front. Plant Sci. 10:117. doi: 10. 3389/fpls.2019.00117

Selsted, M. E., and Ouellette, A. J. (2005). Mammalian defensins in the antimicrobial immune response. Nat. Immunol. 6, 551-557. doi: 10.1038/ni1206

Seyfferth, C., and Tsuda, K. (2014). Salicylic acid signal transduction: the initiation of biosynthesis, perception and transcriptional reprogramming. Front. Plant Sci. 5:697. doi: 10.3389/fpls.2014.00697

Shafee, T. M., Lay, F. T., Hulett, M. D., and Anderson, M. A. (2016). The defensins consist of two independent, convergent protein superfamilies. Mol. Biol. Evol. 33, 2345-2356. doi: 10.1093/molbev/msw106

Shen, Q., Liu, Y., and Naqvi, N. I. (2018). Fungal effectors at the crossroads of phytohormone signaling. Curr. Opin. Microbiol. 46, 1-6. doi: 10.1016/j.mib. 2018.01.006

Siddique, S., Matera, C., Radakovic, Z. S., Hasan, M. S., Gutbrod, P., Rozanska, E., et al. (2014). Parasitic worms stimulate host NADPH oxidases to produce reactive oxygen species that limit plant cell death and promote infection. Sci. Signal. 7:ra33. doi: 10.1126/scisignal.2004777

Smirnoff, N., and Arnaud, D. (2019). Hydrogen peroxide metabolism and functions in plants. New Phytol. 221, 1197-1214. doi: 10.1111/nph.15488

Stephenson, K., and Hoch, J. A. (2002). Virulence-and antibiotic resistanceassociated two-component signal transduction systems of Gram-positive pathogenic bacteria as targets for antimicrobial therapy. Pharmacol. Ther. 93, 293-305. doi: 10.1016/s0163-7258(02)00198-5

Stock, A. M., Robinson, V. L., and Goudreau, P. N. (2000). Two-component signal transduction. Ann. Rev. Biochem. 69, 183-215.

Stotz, H. U., Thomson, J., and Wang, Y. (2009). Plant defensins: defense, development and application. Plant Signal. Behav. 4, 1010-1012. doi: 10.4161/ psb.4.11.9755

Svetaz, L. A., Bustamante, C. A., Goldy, C., Rivero, N., Müller, G. L., Valentini, G. H., et al. (2017). Unravelling early events in the Taphrina deformans-Prunus persica interaction: an insight into the differential responses in resistant and susceptible genotypes. Plant Cell Environ. 40, 1456-1473. doi: 10.1111/pce. 12942

Terras, F. R., Torrekens, S., Van Leuven, F., Osborn, R. W., Vanderleyden, J., Cammue, B. P., et al. (1993). A new family of basic cysteine-rich plant antifungal proteins from Brassicaceae species. FEBS Lett. 316, 233-240. doi: 10.1016/00145793(93)81299-f

Thery, T., and Arendt, E. K. (2018). Antifungal activity of synthetic cowpea defensin Cp-thionin II and its application in dough. Food Microbiol. 73, 111-121. doi: 10.1016/j.fm.2018.01.006

Thevissen, K., Warnecke, D. C., François, I. E., Leipelt, M., Heinz, E., Ott, C., et al. (2004). Defensins from insects and plants interact with fungal glucosylceramides. J. Biol. Chem. 279, 3900-3905. doi: 10.1074/jbc.m311165200

Torres, M. A., Jones, J. D., and Dangl, J. L. (2006). Reactive oxygen species signaling in response to pathogens. Plant Physiol. 141, 373-378. doi: 10.1104/pp.106. 079467

Tuskan, G. A., Difazio, S., Jansson, S., Bohlmann, J., Grigoriev, I., Hellsten, U., et al. (2006). The genome of black cottonwood, Populus trichocarpa (Torr. \& Gray). Science 313, 1596-1604.

Vanholme, R., De Meester, B., Ralph, J., and Boerjan, W. (2019). Lignin biosynthesis and its integration into metabolism. Curr. Opin. Biotechnol. 56, 230-239. doi: 10.1016/j.copbio.2019.02.018

Velivelli, S. L., Islam, K. T., Hobson, E., and Shah, D. M. (2018). Modes of action of a bi-domain plant defensin MtDef5 against a bacterial pathogen Xanthomonas campestris. Front. Microbiol. 9:934. doi: 10.3389/fmicb.2018.00934 
Vriens, K., Cammue, B., and Thevissen, K. (2014). Antifungal plant defensins: mechanisms of action and production. Molecules 19, 12280-12303. doi: 10 . 3390/molecules 190812280

Wang, C., Liu, Y., Li, S. S., and Han, G. Z. (2015). Insights into the origin and evolution of the plant hormone signaling machinery. Plant Physiol. 167, 872-886. doi: 10.1104/pp.114.247403

Waterman, A. M., and Cash, E. K. (1950). Leaf blotch of poplar caused by a new species of Septotinia. Mycologia 42, 374-384. doi: 10.1080/00275514.1950. 12017843

Wingfield, M. J., Brockerhoff, E. G., Wingfield, B. D., and Slippers, B. (2015). Planted forest health: the need for a global strategy. Science 349, 832-836. doi: $10.1126 /$ science.aac6674

Yan, C., and Xie, D. (2015). Jasmonate in plant defence: sentinel or double agent? Plant Biotechnol. J. 13, 1233-1240. doi: 10.1111/pbi.12417

Yang, Y. X., Ahammed, G. J., Wu, C., Yang, Z., Wan, C., and Chen, J. (2018). Red light-induced systemic resistance against root-knot nematode is mediated by a coordinated regulation of Salicylic acid, Jasmonic acid and redox signaling in watermelon. Front. Plant Sci. 9:899. doi: 10.3389/fpls.2018. 00899

Yasuda, M., Ishikawa, A., Jikumaru, Y., Seki, M., Umezawa, T., Asami, T., et al. (2008). Antagonistic interaction between systemic acquired resistance and the abscisic acid-mediated abiotic stress response in Arabidopsis. Plant Cell 20, 1678-1692. doi: 10.1105/tpc.107.054296
Yount, N. Y., and Yeaman, M. R. (2004). Multidimensional signatures in antimicrobial peptides. Proc. Natl. Acad. Sci. U.S.A. 101, 7363-7368. doi: 10. 1073/pnas.0401567101

Zehnder, G. W., Murphy, J. F., Sikora, E. J., and Kloepper, J. W. (2001). Application of rhizobacteria for induced resistance. Eur. J. Plant Pathol. 107, 39-50.

Zhao, M., Ma, Y., Pan, Y. H., Zhang, C. H., and Yuan, W. X. (2011). A hevein-like protein and a class I chitinase with antifungal activity from leaves of the paper mulberry. Biomed Chromatogr. 25, 908-912. doi: 10.1002/bmc

Zhu, L. H., Xu, W., Yang, J. Y., Li, D. W., Ge, J. M., Ye, J. R., et al. (2019). First report of Septotinia populiperda causing leaf blotch of Salix babylonica in China. Plant Dis. 103, 768-769.

Conflict of Interest: The authors declare that the research was conducted in the absence of any commercial or financial relationships that could be construed as a potential conflict of interest.

Copyright (C) 2020 Wei, Movahedi, Xu, Sun, Wang, Li, Yin and Zhuge. This is an open-access article distributed under the terms of the Creative Commons Attribution License (CC BY). The use, distribution or reproduction in other forums is permitted, provided the original author(s) and the copyright owner(s) are credited and that the original publication in this journal is cited, in accordance with accepted academic practice. No use, distribution or reproduction is permitted which does not comply with these terms. 